G. E. Müller: Ueber d. Maassbestimmungen d. Ortssinnes d. Haut etc. 191

\title{
Ueber die Maassbestimmungen des Ortssinnes der Haut mittels der Methode der richtigen und falschen Fälle.
}

\author{
Von- \\ Dr. phil. G. E. Mülller, \\ Privatdocent der Philosophie in Göttingen.
}

I. Von der mathematischen Beziehung, die zwischen den Feinheitsmaassen des Ortssinnes und den erhaltenen Zahlen richtiger und falscher Fälle besteht.

\$. 1.

Der Ortssinn einer Hautgegend gilt als um so feiner, je geringer der Werth der sogenannten Raumschwelle für die gegebene Hautstelle ist. Hierbei wird unter der Raumschwelle derjenige Abstand zweier die Haut gleichzeitig berührender Zirkel- oder Nadelspitzen verstanden, welcher erforderlich ist, damit eben noch der Eindruck einer Berührung an zwei verschiedenen, nicht unmittelbar an einander angrenzenden Hautpunkten entstehe. Die Raumschwelle einer gegebenen Hautgegend besitzt jedoch auch bei ganz gleich bleibenden Versuchsumständen keineswegs immer denselben Werth. Berührt man nämlich ein und dieselbe Hautstelle zu oft wiederholten Malen mit $\mathbf{2}$ gleichzeitig aufgesetzten Zirkelspitzen, deren Abstand immer derselbe bleibt, so wird bekanntermassen bei geeigneter Wahl dieses Spitzenabstandes in unregelmässiger und zufälliger Weise 'das eine Mal der Eindruck einer Doppelberührung, das andere Mal der Eindruck einer einfachen Berïhrung erhalten ${ }^{1}$ ). Dieser Thatbestand scheint zu ergeben,

1) Ausser den Fällen, wo der Eind̊ruck einer einfachen oder einer Doppelberührung entsteht, kommen bekanntlich auch noch unentschiedene Fälle vor, wo das Urtheil über die Zahl der berührenden Spitzen zurückgehalten wird. Indessen sind solche unentschiedene Fälle bei einigen Beobachtern, 
dass die Grösse der Raumschwelle für jede Hautgegend zufälligen Schwankungen unterliegt, in Folge deren ein und derselbe gegebene Spitzenabstand das eine Mal grösser, das andere Mal kleiner sein kann als die Raumschwelle. Den Mittelwerth, um welchen die in den einzelnen Beobachtungsfällen vorhandenen zufälligen Werthe der Raumschwelle herumschwanken, bezeichne ich kurz als den Werth $S$. Offenbar hat dieser Mittelwerth, welcher im Gegensatze zu jenen zufälligen Einzelwerthen der Raumschwelle eine von der Lokalität der untersuchten Hautstelle u. dergl. abhängige constante Grösse repräsentirt, bei Bestimmung der Feinheit des Ortssinnes als Unterlage zu dienen. Die Aufgabe, welche der psychophysischen Methodenlehre aus den im Gebiete des Ortssinnes vorliegenden Anwendungen der Methode der richtigen und falschen Fälle erwächst, besteht demnach vor Allem darin, anzugeben, in welcher Weise der Werth $S$, d. h. der Mittelwerth der zufällig schwankenden Grössen der Raumschwelle, aus den experimentell erhaltenen Zahlen richtiger und falscher Fälle zu berechnen sei; wobei als richtige Fälle diejenigen gelten, in denen die beiden berïhrenden Zirkel- oder Nadelspitzen den Eindruck einer Doppelberührung hervorrufen, als falsche Fälle hingegen diejenigen, in denen der Eindruck einer einfachen Berührung entsteht.

\section{§. 2.}

Nach Vorstehendem ist die Raumschwelle in einem gegebenen Beobachtungsfalle um eine zufällige Grösse, die wir als den zufälligen Fehler $\pm \delta$ bezeichnen, grösser oder kleiner als der Werth $S$. Bezeichnen wir daher die thatsächliche Distanz der beiden gleichzeitig aufgesetzten Nadel- oder Zirkelspitzen mit $D$, so wird der Eindruck einer Doppelberïhrung immer nur dann eintreten, wenn $D>S \pm \delta$ ist. Führt man also mit constantem $D$ eine hinreichend grosse Anzahl von Versuchen an einer gegebenen Hautstelle aus, so wird das Verhältniss der Zahl $r$ der richtigen Fälle zur Gesammtzahl $n$ der Fälle gleich der Wahrscheinlichkeit oder rela-

nämlich bei Kottenkamp und Ullrich, Volkmann und während zweier Versuchsreihen auch bei Hartmann gar nicht vorgekommen. Ich gehe demgemäss im Obigen zunächst von der Betrachtung des einfacheren Falles aus, wo über die Zahl der berührenden Spitzen immer ein bestimmtes Urtheil gefällt wird. 
tiven Häufigkeit derjenigen Fälle sein, in denen $D>S \pm \delta$ ist. Falls $D>S$ ist, muss $D>S \pm \delta$ sein, erstens in allen denjenigen Fällen, in denen $\delta$ negativ ist, also das untere Vorzeichen von $\delta$ gilt, und zweitens in denjenigen Fällen, in denen $\delta$ positiv und $<D-S$ ist. Nehmen wir an, dass für die Fehler $\pm \delta$ das vielfach bewährte Gauss'sche Fehlergesetz gültig sei, so ist die Wahrscheinlichkeit der ersteren Fälle $=1 / 2$ und diejenige der zweiten Fälle $=\frac{h}{\sqrt{\pi}} \int_{o}^{\frac{D}{D}} e^{-h^{2} \delta^{2}} d \delta$, wo $h$ das sogenannte Präcisionsmaass darstellt und $=\frac{1}{\delta_{m} \sqrt{\pi}}$ zu setzen ist, wenn wir mit $\delta_{m}$ den mittleren Werth der Fehler $\pm \delta$ bezeichnen. Es ist mithin, falls $D>S$ und demgemäss $\frac{r}{n}>1 / 2$ ist,

$$
\frac{r}{n}=\frac{1}{2}+\frac{h}{\sqrt{\pi}} \int^{c D-S} e^{-h^{2} \delta^{2}} d \delta
$$

oder, wenn wir $h \delta=t$ setzen,

$$
\frac{r}{n}=\frac{1}{2}+\frac{1}{\sqrt{\pi}} \int_{0}^{a(D-S) h} e^{-t^{2}} d t
$$

Für den Fall, dass $D<S$ ist, kann $D$ nur dann $>S \pm \delta$ sein, wenn $\delta$ negativ und seinem absoluten Werthe nach grösser als $S-D$ ist. Mithin ist, falls $D<S$ und demgemäss $\frac{r}{n}<1 / 2$ ist,

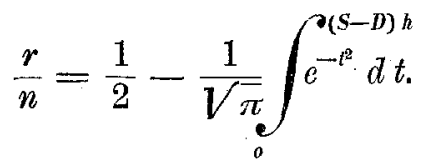

Für den Fall, dass $\frac{r}{n}=1 / 2$ ist, muss, wie leicht zu erkennen, $S=D$ gesetzt werden.

Was die Zahl $f$ der falschen Fälle betrifft, in denen $D<S \pm \delta$ ist und in Folge dessen der Eindruck einer einfachen Berührung

1) Obige Formeln (1) und (2) hat auch Herr Prof. Fechner gefunden, von welchem eine über diesen Gegenstand mit handelnde Abhandlung in Aussicht steht. 
entsteht, so ergibt sich die Beziehung des Quotienten $\frac{f}{n}$ zu $S, h$ und $D$ unmittelbar aus dem Vorstehenden, da ja $\frac{f}{n}=1-\frac{r}{n}$ ist.

§. 3.

Im Bisherigen sind nur diejenigen Versuchsreihen berïcksichtigt, in denen blos richtige oder falsche, aber nicht auch unentschiedene Fälle erhalten worden sind. Indessen es bedarf nur einer gewissen Erweiterung der obigen Betrachtungsweise, um auch denjenigen Versuchsreihen (von Paulus, Riecker und Hartmann) genügen zu können, in denen ausser den richtigen und falschen Fällen auch noch unentschiedene Fälle vorkamen. Wir bezeichnen als Raumschwelle oder als Sehwelle der richtigen Fälle denjenigen Werth, welchen der Abstand zweier gleichzeitig aufgesetzter Zirkel- oder Nadelspitzen besitzen muss, um eben den richtigen Eindruek einer Doppelberiihrung za bewirken; und wir sind im Bisherigen von der Voranssetzung ausgegangen, dass jeder Spitzenabstand, der kleiner sei als die Schwelle der richtigen Fälle, nothwendig den Eindruck einer einfachen Berührung hervorrufen mïsse. Die Thatsache der unentschiedenen Fälle zeigt nun, dass letztere Voraussetzung nicht allgemein erfüllt ist, dass vielmehr neben jener Schwelle der richtigen Fälle noch eine Schwelle der unentschiedenen Fälle anzunehmen ist, welche einen geringeren Werth besitzt als die Schwelle der richtigen Fälle und dadurch charakterisirt ist, dass jeder Spitzenabstand, welcher kleiner ist als die Schwelle der unentschiedenen Fälle, den Eindruck einer einfachen Berührung bewirkt, hingegen jeder Spitzenabstand, welcher grösser ist als diese Schwelle, entweder ein nnentschiedenes Urtheil zur Folge hat oder den Eindruck einer Doppelberihrung hervorruft, und zwar ersteres dann, wenn der Spitzenabstand zwar grösser ist als die Schwelle der unentschiedenen Fälle, aber die Schwelle der richtigen Fälle noch nicht erreicht, letzteres dann, wenn der Spitzenabstand nicht blos die erstere, sondern auch die letztere Schwelle iibersteigt. Stellt also

der Abstand $A C$ die Schwelle der richtigen Fälle dar, so
wird die Schwelle der unentschiedenen Fälle $<A C$, etwa
$=A B$ sein; und das Urtheil wird immer dann ein falsches sein,


wenn der Spitzenabstand $D<A B$ ist; ein unentschiedener Fall wird eintreten, wenn $D>A B$, aber $<A C$ ist; der richtige Eindruck einer Doppelberiihrung endlich wird entstehen, wenn $D>A C$ ist.

Da der Erfahrung gemäss ein und derselbe Spitzenabstand bei Berührung einer und derselben Hautstelle in unregelmässiger und zufälliger Weise das eine Mal einen unentschiedenen Fall, das andere Mal einen richtigen oder falschen Fall zur Folge haben kann, so ergibt sich, dass ebenso wie die Schwelle der richtigen Fälle auch diejenige der unentschiedenen Fälle zufälligen Schwankungen unterliegt ${ }^{1}$ ). Bezeichnen wir wie bisher den Mittelwerth der Schwelle der richtigen Fälle mit $S$, so können wir den Werth, um welchen die zufälligen Grössen der Schwelle der unentschiedenen Fälle herumschwanken, kurz mit $S^{\prime}$ bezeichnen. Dieser Mittelwerth der Schwelle der unentschiedenen Fälle repräsentirt im Gegensatze zu den von Zufälligkeiten abhängigen Einzelwerthen dieser Schwelle eine in gewissem Sinne constante Grösse und kann ebenso wie der Werth $S$ bei Bestimmung der Feinheit des Ortssinnes als Unterlage dienen. Die Beziehung, in welcher die Werthe $S$ und $S^{\prime}$ zu den experimentell erhaltenen Zahlen richtiger und falscher Fälle stehen, lässt sich nach dem Früheren leicht angeben.

Auch dann nämlich, wenn neben den richtigen und falschen Fällen noch unentschiedene Fälle vorkommen, ist der Erfahrung gemäss die Schwelle der richtigen Fälle eine zufällig schwankende und daher allgemein $=S \pm \delta$ zu setzen, wo $\delta$ eine zufällig variable Grösse bedentet. Die Zahl der richtigen Fälle ist demgemäss mit der Zahl derjenigen Fälle identisch, in denen $D>S \pm \delta$ ist, und es gelten mithin (vergl. S. 193) betreffs des Verhältnisses $\frac{r}{n}$ auch dann, wenn unentschiedene Fälle mit vorkommen, noch die obigen Gleichungen (1) und (2).

Was das Verhältniss $\frac{f}{n}$ betrifft, das beim Vorkommen unent-

1) Im Allgemeinen hat mạn sich vorzustellen, dass, wenn die Schwelle der richtigen Fälle eine positive oder negative zufällige Schwankung erfährt, alsdann immer auch die Schwelle der unentschiedenen Fälle eine zufällige Schwankung nach derselben Richtung hin erfährt, dass also immer dann, wenn in obigem Beispiele sich der Punkt $C$ dem Punkte $A$ nähert oder davon entfernt, auch $B$ sich dem $A$ nähert, bez. davon entfernt. 
schiedener Fälle nicht mehr $=1-\frac{r}{n}$ zu setzen ist, so ist die Schwelle der unentschiedenen Fälle als eine zufällig schwankende allgemein $=S^{\prime} \pm \delta^{\prime}$ zu setzen, wo $S^{t}$ wie oben den Mittelwerth dieser Schwelle und $\delta^{\prime}$ eine zufällig variable Grösse bedeutet, mithin nach Obigem die Zahl der falschen Fälle mit der Zahl derjenigen Fälle identisch, in denen $D<S^{*} \pm \delta^{*}$ ist. Es ist also das Verhältniss $\frac{f}{n}$ bei hinlänglich grosser Versuchszahl gleich der Wahrscheinlichkeit, dass $D<S^{\prime} \pm \delta^{4}$ sei. Hieraus ergibt sich, falls, $D<S^{\prime}$ und demgemäss $\frac{f}{n}>1 / 2$ ist,

$$
\frac{f}{n}=\frac{1}{2}+\frac{1}{\sqrt{\pi}} \int_{\substack{g \\ o}}^{\partial\left(S^{2}-D\right) h^{\prime}} e^{-t^{2}} d t
$$

und, falls $D>S^{4}$ und demgemäss $\frac{f}{n}<1 / 2$ ist,

$$
\frac{f}{n}=\frac{1}{2}-\frac{1}{\sqrt{\pi}} \int_{0}^{\left(D-S^{\prime}\right) h^{t}} e^{-t^{2}} d t
$$

wo das Präcisionsmaass $h^{\prime}$ gleich $\frac{1}{\delta^{i_{m}} \sqrt{\pi}}$ zu setzen ist, wenn wir mit $\delta_{m}{ }_{m}$ den mittleren Werth der Fehler $\delta^{\prime}$ bezeichnen. Falls $\frac{r}{n}=1 / 2$ erhalten wird, ist $D=S$ und, falls $\frac{f}{n}=1 / 2$ ausfällt, $D=S^{\prime}$ zu setzen.

Die Gleichungen (3) und (4) lassen sich auch dann als giultig betrachten, wenn gar keine unentschiedenen Fälle vorkommen. Es ist dann $S^{\prime}=S$ und demgemäss auch $h^{*}=h$ zu setzen. Die Addition der Gleichungen (1) und (4), (2) und (3) ergiebt alsdann $\frac{r}{n}+\frac{f}{n}=1$. 
II. Von den Verfahrungsweisen, nach denen sich die Feinheitsmáasse des Ortssinnes und die Präcisionsmasse aus den Versuchsresultaten ableiten lassen.

\section{§. 4 .}

Nachdem wir im Vorstehenden die mathematische Beziehung, welche zwischen $\frac{r}{n}$, bez. $\frac{f}{n}$, einerseits und $h$ und $S$, bez. $h^{\prime}$ und $S^{\prime}$, andrerseits besteht, festgestellt haben, gehen wir nun zu den Verfahrungsweisen über, nach denen sich auf Grund obiger 4 Fundamentalgleichungen aus den experimentell erhaltenen Verhältnissen $\frac{r}{n}$, bez. $\frac{f}{n}$, die thatsächlichen Werthe von $h$ and $h, S$ und $S^{4}$ und mithin auch die Feinheitsmaasse des Ortssinnes $\frac{1}{S}$ und $\frac{1}{S^{\prime \prime}}$ berechnen lassen. Und zwar wird es genügen, die Verfahrungsweisen, nach denen $h$ und $S$ auf Grund der Gleichungen (1) und (2) aus den erhaltenen Verhältnissen $\frac{r}{n}$ zu berechnen sind, näher zu erörtern, da die Art and Weise, wie eventuell $h^{\prime}$ und $S^{t}$ auf Grund der Gleichungen (3) und (4) aus den Verhältnissen $\frac{f}{n} \mathrm{zu}$ bestimmen sind, mit der Art der Berechnung von $h$ und $S$ aus den Verhältnissen $\frac{r}{n}$ offenbar identisch ist.

Setzen wir kurz $(D-S) h=\tau$ und $(S-D) h=-\tau$, so lässt sich, falls man nur mit einem einzigen Spitzenabstande $D$ eine Reihe von Versuchen ausgeführt hat, nach Gleichung (1), bez. (2), aus dem erhaltenen Verhältnisse $\frac{r}{n}$ zwar ein bestimmter zugehöriger Werth von $\tau$ ableiten, nicht aber auch die Grösse von $S$, weil in dem Ausdrucke für $\tau$ ausser $S$ auch noch $h$ eine unbekannte Grösse ist. Hat man jedoch mit zwei verschiedenen Spitzenabständen $D_{1}$ and $D_{2}$ operirt und dem entsprechend 2 verschiedene, mit $\frac{r_{1}}{n}$ und $\frac{r_{2}}{n}$ zu bezeichnende Werthe von $\frac{r}{n}$ gefunden, so erhält man 
mittels Gleichung (1), bez. (2), auch 2 verschiedene Werthe von $\left.\tau^{1}\right)$, nämlich $\tau_{1}$ und $\tau_{2}$, und zwar ist

$$
\text { und } \begin{aligned}
\tau_{1} & =\left(D_{1}-S\right) h \\
\tau_{2} & =\left(D_{2}-S\right) h .
\end{aligned}
$$

Auf Grund dieser beiden Gleichungen, in denen ausser $\tau_{1}$ and $\tau_{2}$ auch $D_{1}$ und $D_{2}$ bekannt sind, berechnen sich dann in einfacher Weise die beiden Unbekannten $h$ und $S$.

Hat man eine und dieselbe Hautstelle mit mehr als 2 verschiedenen Spitzenabständen untersucht, also mehr als 2 Gleichungen zur Bestimmung von $h$ und $S$ erbalten, so muss die Methode der kleinsten Quadrate Anwendung finden, und zwar gestaltet sich eine genaue Befolgung der ïblichen Vorschriften derselben im vorliegenden Falle folgendermassen. Man sucht zunächst auf irgend welchem Wege möglichst genaue Näherungswerthe von $h$ und $S$ zu erhalten, etwa dadurch, dass man aus 2 der beobachteten Verhältnisse $\frac{r}{n}$, welche anscheinend besonders gut ausgefallen sind, in der soeben angegebenen Weise $h$ und $S$ berechnet. Betreffs des Näherungswerthes von $S$ bedarf es an und für sich meist gar keiner weiteren Berechnung, da sich fast stets der Tabelle der Versuchsresultate ohne Weiteres entnehmen lässt, bei welchem Spitzenabstande ungefähr $\frac{r}{n}=1 / 2$ ausgefallen, d. h. $D=S$ gewesen sein

1) Wie leicht zu erkennen, ist der Werth von $\tau$ negativ, wenn $\frac{r}{n}<1 / 2$ ist. - Behufs Ableitung der Werthe von $\tau$ aus den Verhältnissen $\frac{r}{n}$ bedient man sich am besten der von Fechner (Elemente der Psychophysik I, S. 108 f.) gegebenen Fundamentaltabelle der Nethode der richtigen und falschen Fälle und der dazu gehörigen Zusatztabellen, wo $\tau$ unter der Bezeichnung $t=h D$ aufgeführt ist. Falls $\frac{r}{x}<1 / 2$ ist, bestimmt man mittels dieser Tabellen den entsprechenden Werth von $t$ in der Weise, dass man nach Gleichung (1) für $1-\frac{r}{n}$ den zugehörigen Werth von $r$ aufsucht und demselben ein negatives Vorzeichen gibt. Die Richtigkeit dieses Verfahrens erhellt daraus, dass, falls $\frac{r}{n}<1 / 2$ ist, nach Gleichung (2) $1-\frac{r}{n}=\frac{1}{2}+\frac{1}{\sqrt{\pi}} \int_{0}^{(S-D) h} e^{-l^{2} d t}$ ist. 
würde. Hat man auf diesem oder jenem Wege Näherungswerthe von $h$ und $S$ erhalten, die mit $h_{0}$ und $S_{0}$ bezeichnet werden mögen, so setze man allgemein, falls $\frac{r}{n}>1_{1}^{\prime}$ ist,

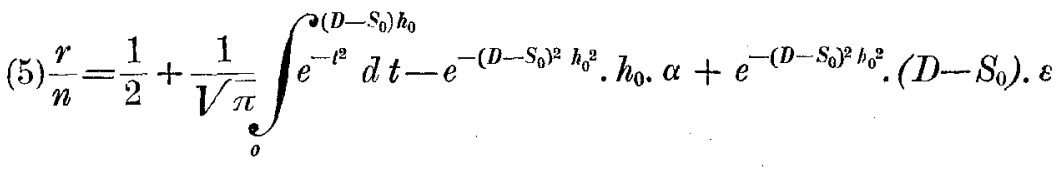
und, falls $\frac{r}{n}<1 / 2$ ist,

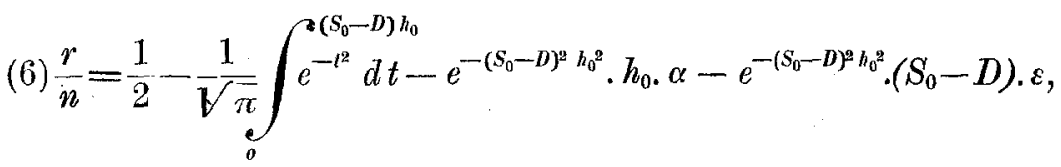
wo $\alpha$ und $\varepsilon$ die erst zu bestimmenden plausibelsten Verbesserungen der eingeführten Näherungswerthe $S_{0}$ und $h_{0}$ bedeuten. Auf Grund dieser allgemeinen Gleichungen erhält man durch Einsetzen der benutzten Werthe von $D$ und der beobachteten Werthe von $\frac{r}{n}$ eine Reihe specieller Gleichungen, deren Zahl gleich der Zahl der benutzten Spitzenabstände und der erhaltenen Verhältnisse $\frac{r}{n}$ ist, und in deren jeder (abgesehen von dem Falle, wo $D=S_{0}$ ist) beide Unbekannte $\alpha$ und $\varepsilon$ auftreten und zwar nur als lineare Funktionen der Beobachtung:sgrössen vorkommen. Aus letzteren Gleichungen berechnet man darauf durch Bildung der Normalgleichungen ${ }^{1}$ ) die

1) Bestehen zwischen den Beobachtungsgrössen $l_{1}, l_{2}, l_{3}$ u. s. f., deren Gewichte $g_{1}, g_{2}, g_{3}$ u. s. f. sein mögen, und den beiden Unbekannten $x$ und $y$ die Gleichungen:

$$
\begin{gathered}
l_{1}=a_{1} x+b_{1} y \\
l_{2}=a_{2} x+b_{2} y \\
l_{3}=a_{3} x+b_{3} y \\
\text { u. s. f. }
\end{gathered}
$$

wo $a_{1}, a_{2}$ u. s. f., $b_{1}, b_{2}$ u. s. f. bekannte Coefficienten der Unbekannten sind, so werden als Normalgleichungen folgende, zur Bestimmung von $x$ und $y$ dienende Gleichungen bezeichnet:

$$
\begin{aligned}
& {[a a g] x+[a b g] y=[a l g]} \\
& {[a b g] x+[b b g] y=[b l g]}
\end{aligned}
$$

wo die eckige Klammer als Summenzeichen dient, mithin [aag] die Summe $a_{1} a_{1} g_{3}+a_{2} a_{2} g_{2}+a_{3} a_{3} g_{3}$ u.s. f. bedeutet, $[a b g]$ die Summe $a_{1} b_{1} g_{1}+a_{2} b_{2} g_{2}+$ $a_{3} b_{3} g_{3}$ u. s. f. darstellt, u. s. w. 
Werthe von $\alpha$ und $\varepsilon$, und indem man den gefundenen Werth von $\alpha$ zu dem Näherungswerthe $S_{0}$ und den gefundenen Werth von $\varepsilon$ zu dem Näherungswerthe $h_{0}$ algebraisch addirt, erhält man in $S_{0}+\alpha$ und in $h_{0}+\varepsilon$, wo $\alpha$ und $\varepsilon$ negativ sein können, die plausibelsten Bestimmungen von $S$ und $h$. Hierbei ist jedoch vorausgesetzt, dass bereits die eingeführten Näherungswerthe $S_{0}$ und $h_{0}$ auf ziemlich genaue Weise bestimmt worden und mithin die Verbesserungen $\alpha$ und $\varepsilon$ nur sehr klein ausgefallen sind, so dass die zweiten und höheren Potenzen derselben als ganz verșehwindend gelten können. Ist Letzteres nicht der Fall, so muss die Rechnung noch weiter geführt werden, indem man die erhaltenen Werthe $S_{0}+\alpha$ und $h_{0}+\varepsilon$ als blosse Näherungswerthe betrachtet und für dieselben ganz in der obigen Weise die plausibelsten Verbesserungen $\alpha_{1}$ und $\varepsilon_{1}$ bestimmt.

\section{\$. 5 .}

Wie man leicht erkennt, ist eine Befolgung des vorstehenden, kurz als Verfahren I za bezeichnenden Verfahrens, das genau den Vorschriften der Methode der kleinsten Quadrate entspricht, eine sehr langwierige und mühsame. Die Berechnung von $h$ und $S$ aus einer einzigen Versuchsreihe kann nach diesem Verfahren leicht einen ganzen Tag beanspruchen. Weit einfacher und bequemer ist ein kurz als Verfahren II zu bezeichnendes Verfahren, bei dem man nicht, wie streng genommen geschehen muisste, auf die Werthe von $\frac{r}{n}$ als unmittelbar beobachtete Grössen zurückgeht, sondern die aus den Verhältnissen $\frac{r}{n}$ nach Gleichung (1), bez. (2), abgeleiteten Werthe von $\tau$ als unmittelbar beobachtete Grössen betrachtet. Nach diesem Verfahren bestimmt man zu jedem der erhaltenen Verhältnisse $\frac{r_{1}}{n}, \frac{r_{2}}{n}$ u. s. f. den zugehörigen Werth $\tau_{1}, \tau_{2}$ u. s. f. und setzt alsdann

$$
\begin{gathered}
h\left(D_{1}-S\right)=\tau_{1} \\
h\left(D_{2}-S\right)=\tau_{2} \\
h\left(D_{3}-S\right)=\tau_{3} \\
\text { u. s. f. }
\end{gathered}
$$

oder, indem man $h S=k$ setzt 


$$
\begin{aligned}
& h \dot{D}_{1}-k=\tau_{1} \\
& h D_{2}-\hbar=\tau_{2} \\
& h D_{3}-\hbar=\tau_{3}
\end{aligned}
$$

u. s. f.

Auf Grund letzterer Gleichungen, in denen die Spitzenabstände $D_{1}, D_{2}$ u. s. f. bekannt sind und die gleichfalls bekannten Werthe $\tau_{1}, \tau_{2}$ u. s. f. als unmittelbar beobachtete Grössen gelten, berechnet man in einfacher Weise durch Bildung der Normalgleichungen (vgl. die Anmerkung zu S. 199) die Werthe von $h$ und $k$. Aus dem berechneten Werthe von $k$ ergibt sich endlich durch Division mit $h$ der gesuchte Mittelwerth der Raumschwelle, die Grösse $S$. Liegt den verschiedenen beobachteten Werthen von $\frac{r}{n}$ nicht ein und dieselbe Versuchszahl $n$ zu Grunde, so hat man selbstverständlich, falls die Unterschiede der Versuchszahlen $n$ beträchtliche sind, das daraus entspringende verschiedene Gewicht der beobachteten Verhältnisse $\frac{r}{n}$ oder vielmehr der daraus abgeleiteten Werthe $\boldsymbol{r}$ bei Bildung der Normalgleichungen mit in Rücksicht zu ziehen.

$$
\text { §. } 6 .
$$

Das Verfahren II besitzt allerdings vor dem Verfahren I ganz unverkennbar den Vorzug weit grösserer Einfachheit und Bequemlichkeit. Leider ist dasselbe jedoch nicht ein richtiges und genaues Verfahren, insofern dabei nicht die wirklich unmittelbar beobachteten Verhältnisse $\frac{r}{n}$, sondern die daraus abgeleiteten Werthe $\tau$ als unmittelbar beobachtete Grössen betrachtet werden. Es drängt sich daher die Frage auf, ob es nicht eine einfache Modification des Verfahrens II gibt, welche wenigstens annähernd die Bequemlichkeit dieses Verfahrens mit der Genauigkeit des Verfahrens I vereint. Um diese Frage beantworten zu können, untersuchen wir zunächst näher, worauf eigentlich der Mangel des Verfahrens II hinausläuft, d. h. welchen Einfluss es haben muss, wenn man bei Bestimmung von $h$ und $S$ nicht auf die Werthe von $\frac{r}{n}$ selbst, sondern auf die daraus abgeleiteten Werthe $\tau$ zuriickgeht, gleich als ob diese letzteren Werthe die unmittelbar beobachteten Grössen seien. 
Unter der (im Folgenden zunächt allgemein beibehaltenen) Voraussetzung, dass allen beobachteten Verhältnissen $\frac{r}{n}$ eine und dieselbe Versuchszahl $n$ zu Grunde liege und mithin allen dieselbe Genauigkeit und dasselbe Gewicht beizulegen sei, besteht unsere Aufgabe bei Berechnung von $h$ und $S$ darin, diese Werthe so zu bestimmen, dass die Summe der Quadrate der Fehlergrössen, um welche die aus $h$ und $S$ rückwärts wieder berechneten Werthe von $\frac{r}{n}$ von den thatsächlich beobachteten Werthen dieses Verhältnisses abweichen, so klein als möglich sei. Bei Benutzung des Verfahrens II bestimmen wir jedoch thatsächlich $h$ und $S$ so, dass die Summe der Quadrate derjenigen Grössen, um welche die aus $h$ und $S$ rückwärts wieder berechneten Werthe von $x$ von den aus den beobachteten Verhältnissen $\frac{r}{n}$ direkt abgeleiteten Werthen von $\tau$ abweichen, ein Minimum wird. Bezeichnen wir die aus den beobachteten Verhältnissen $\frac{r}{n}$ direkt abgeleiteten Werthe von $\tau$ kurz als die beobachteten Werthe von $\tau$, so wird also, kurz gesagt, nach Verfahren II $h$ und $S$ so bestimmt, dass nicht, wie unbedingt geschehen müsste, die Summe der Quadrate der Abweichungen zwischen beobachtetem und berechnetem $\frac{r}{n}$, sondern vielmehr die Summe der Quadrate der Abweichungen zwischen beobachtetem und berechnetem $\tau$ ein Minimum wird. Nun entspricht bei diesem Verfahren dem zwischen $\frac{r}{n}$ and $v$ bestehenden funktionellen Verhältnisse gemäss einer bestimmten Abweichung zwischen beobachtetem und berechnetem $v$ eine um so geringere Abweichung zwischen beobachtetem und berechnetem $\frac{r}{n}$, je mehr das beobachtete $\frac{r}{n}$ sich nach oben oder nach unten hin von $1 / 2$ entfernt. So ist z. B. die Differenz zwischen beobachtetem und berechnetem $\frac{r}{n}$, welcher eine Differenz 0,015 zwischen beobachtetem und berechnetem $\tau$ entspricht, $=0,008,=0,004,=0,001$, je nachdem das beobachtete $\frac{r}{n}$ $=0,632,=0,888,=0,980$ ist. Mithin werden bei Anwendung. des Verfahrens II, bei welchem gleich grosse Abweichungen zwi- 
schen beobachtetem und berechnetem $\tau$, mögen sie nun von $1 / 2$ weit abstehenden oder davon wenig verschiedenen Beobachtungswerthen von $\frac{r}{n}$ angehören, als völlig gleichwiegend betrachtet werden, die Grössen $h$ und $S$ offenbar so bestimmt, dass kleinere Abweichungen zwischen beobachtetem and berechnetem $\frac{r}{n}$, welche weiter von $1 / 2$ abstehenden Werthen von $\frac{r}{n}$ zugehören, als gleichwiegend betrachtet werden wie grössere Abweichungen zwischen beobachtetem and berechnetem $\frac{r}{n}$, welche mittleren, am $1 / 2$ herumliegenden Werthen von $\frac{r}{n}$ entsprechen. Der Mangel des Verfahrens II kommt also kurz darauf hinaus, dass dasselbe beobachteten Werthen von $\frac{r}{n}$, denen thatsächlich gleiches Gewicht zukommt, verschiedenes Gewicht beilegt und zwar so, dass ein erhaltener Werth von $\frac{r}{n}$ mit um so höherem Gewichte in Rechnung kommt, je weiter er sich nach oben oder nach unten hin von dem Werthe $1 / 2$ entfernt.

Nach Vorstehendem muss sich der Unzulänglichkeit des Verfahrens II ganz einfach dadurch abhelfen lassen, dass man dieses Verfahren in der obigen Weise zur Anwendung bringt, aber dabei den beobachteten Verhältnissen $\frac{r}{n}$ ein um so geringeres Gewicht $r$ beilegt, je weiter das beobachtete $\frac{r}{n}$ nach oben oder nach unten hin von $1 / 2$ abweicht, und zwar so, dass die falschen Gewichte, welche nach Obigem die Anwendung des Verfahrens II an und für sich den Beobachtungswerthen von $\frac{r}{n}$ beilegt, durch die Gewichte $I^{*}$ ganz oder wenigstens annähernd compensirt werden. Aus folgender Tabelle I kann man sich entweder unmittelbar oder mittels einfacher Interpolation die Gewichte $I$ entnehmen, die man (unter der Voraussetzung, dass allen benutzten Spitzenabständen $D$ die gleiche Versuchszahl $n$ entspreche) den verschiedenen experimentell erhaltenen Verhältnissen $\frac{r}{n}$ beizulegen hat, wenn man das bequeme Verfahren II anwenden und zugleich der Ungenauig- 
keit desselben in geeigneter Weise abhelfen will. Diejenigen Werthe von $\frac{r}{n}$, welche $<1 / 2$, sind in die Tabelle nicht mit aufgenommen, weil denselben gleiche Gewichte $I$ beizulegen sind wie denjenigen Werthen von $\frac{r}{n}$, welche gleich weit nach oben hin von $1 / 2$ abstehen. Sind also z. B. 4 verschiedene Spitzenabstände zur Anwendung gekommen und für $\frac{r}{n}$ die 4 verschiedenen Werthe $0,25,0,48,0,75$, 0,99 gefunden worden, so hat man dem ersten und dritten dieser Werthe das Gewicht 0,634, dem zweiten das Gewicht 0,997 und endlich dem vierten das Gewicht 0,004 beizulegen und dann $h$ und $S$ einfach nach dem Verfahren II zu berechnen. Ich bezeichne dieses neue, indessen nur durch jene Gewichtscorrektionen von Verfahren II verschiedene, genaue und doch zugleich wenig umständliche Verfahren kurz als das Verfahren III oder das Verfahren mit Gewichtscorrektionen.

Tabelle I.

\begin{tabular}{|c|c|c|c|c|c|c|c|}
\hline$\frac{r}{n}$ & $r$ & $\frac{r}{n}$ & $T$ & $\frac{r}{n}$ & $\Gamma$ & $\frac{r}{n}$ & $\Gamma$ \\
\hline 0,50 & 1 & 0,67 & 0,824 & 0,84 & 0,373 & 0,954 & 0,059 \\
\hline 0,51 & 0,999 & 0,68 & 0,803 & 0,85 & 0,342 & 0,958 & 0,050 \\
\hline 0,52 & 0,997 & 0,69 & 0,782 & $\{0,86$ & 0,311 & 0,962 & 0,043 \\
\hline 0,53 & 0,994 & 0,70 & 0,760 & 0,87 & 0,281 & 0,966 & 0,036 \\
\hline 0,54 & 0,990 & 0,71 & 0,737 & 0,88 & 0,251 & 0,970 & 0,029 \\
\hline 0,55 & 0,984 & 0.72 & 0,712 & 0,89 & 0,222 & 0,973 & 0,024 \\
\hline 0,56 & 0,977 & 0,73 & 0.687 & 0,90 & 0,193 & 0,976 & 0,020 \\
\hline 0,57 & 0,969 & $0, \overline{7} 4$ & 0,661 & 0,905 & 0,180 & 0,979 & 0,016 \\
\hline 0,58 & 0.960 & 0,75 & 0,634 & 0,910 & 0.166 & 0,982 & 0,012 \\
\hline 0,59 & 0,950 & 0,76 & 0,606 & 0,915 & 0,152 & 0,985 & 0,009 \\
\hline 0,60 & 0,938 & 0,77 & 0,578 & 0,920 & 0,139 & 0,988 & 0,006 \\
\hline $0,61$. & 0,925 & 0,78 & 0,550 & 0,925 & 0,126 & 0,990 & 0,004 \\
\hline 0,62 & 0,911 & 0,79 & 0,521 & 0,930 & 0,114 & 0,992 & 0,003 \\
\hline 0,63 & 0,890 & 0,80 & 0,492 & 0,935 & 0,101 & 0,994 & 0,002 \\
\hline 0,64 & $.0,880$ & 0,81 & 0,463 & 0,940 & 0,089 & 0,996 & 0,001 \\
\hline 0,65 & 0,862 & 0,82 & 0,433 & 0,945 & 0,07 & & \\
\hline 0,66 & 0,843 & 0,83 & 0,403 & 0,950 & 0,067 & & \\
\hline
\end{tabular}

Die Regel, nach welcher die' Gewichte $\Gamma$ der verschiedenen Verhältnisse $\frac{r}{n}$ zu berechnen sind, und welche der vorstehenden (übrigens auch für anderweite psychophysische Untersuchungen dienlichen) Tabelle zu Grunde liegt, ergibt sich folgendermaassen. Ist $\frac{r}{n}$ der beobachtete und $\frac{r}{n} \pm d$ der aus den 
gefundenen Werthen von $h$ und $S$ rückwärts wieder berechnete Werth der relativen $\mathrm{Zahl}$ der richtigen Fälle und bezeichnen wir mit $\tau$ den $\mathrm{zu} \frac{r}{n}$ und mit $x \pm \lambda$ den zu $\frac{r}{n} \pm d$ nach Gleichung (1), bez. (2), zugehörigen Werth von $(D-S) h$, bez. $(S-D) h$, so ist nach diesen Gleichungen

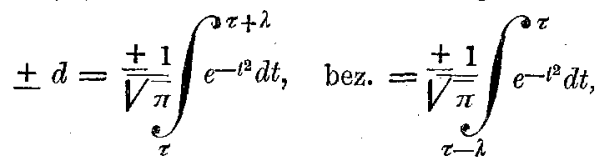

oder, wenn wir die zweite und die höheren Potenzen von $\lambda$ ganz vernachlässigen,

$$
\pm d=\frac{ \pm e^{-\tau^{2}} \cdot \lambda}{\sqrt{\pi}}
$$

Da nun bei Anwendung des Verfahrens II $h$ und $S$ so bestimmt werden, dass $\left[\lambda^{2} 7^{1}\right.$ ) und mithin auch $\left[\frac{\lambda^{2}}{\pi}\right]$ ein Minimum wird, so wird also nach diesem Verfahren, da $\frac{\lambda^{3}}{\pi}=d^{2} \cdot e^{2} \tau^{2}$ ist, $h$ und $S$ so bestimmt, dass nicht $\left[d^{2}\right]$, wie umbedingt geschehen müsste, sondern vielmehr $\left[d^{2} \cdot e^{2} \tau^{2}\right]$ ein Minimum wird. Käme jedem beobachteten $\frac{r}{n}$ ein Gewicht $z u$, welches $=e^{2 z^{2}}$ ist, so müsste allerdings $h$ und $S$ so bestimmt werden, dass $\left[d^{2} \cdot e^{2} x^{2}\right]$ ein Minimum wird. Folglich werden nach Verfahren II $h$ und $S$ so bestimmt, als käme jedem $\frac{r}{n}$ ein Gewicht $e^{2 \tau^{2}} \mathrm{zu}$; der Ungenauigkeit dieses Verfahrens muss sich daher einfach dadurch abhelfen lassen, dass man bei Anwendung desselben jedem $\frac{r}{n}$ ein Gewicht $e^{-2 \tau^{2}}$ beilegt. Die Gewichte $T$ obiger Tabelle sind demgemäss einfach in der Weise ermittelt worden, dass $\Gamma=e^{-2 \tau^{2}}$ gesetzt wurde, wo $\tau$ den aus dem zugehörigen $\frac{r}{n}$ nach Gleichung (1) ableitbaren Werth von $(D-S) h$ bezeichnet. Ob das Verfahren III oder das weit umständlichere Verfahren I zu genaueren Resultaten führt, hängt einerseits von der Zahl der Decimalstellen ab, die man bei Bestimmung jener Gewichte $\Gamma$ berücksichtigt ${ }^{2}$ ), andererseits von der Genauigkeit der Näherungswerthe, welche bei Anwendung

1) Einem häufigen Gebrauche folgend benutze ich die eckige Klammer als Summenzeichen.

2) In Tabelle I habe ich mich auf 3 Decimalstellen beschränkt. Es kann jedoch in Ausnahmefällen (z. B. dann, wenn ein Beobachtungswerth von $\frac{r}{n}$ grösser als 0,996 ausgefallen ist) nothwendig sein, eine grössere Anzahl von Decimalstellen zu berücksichtigen. 
des Verfahrens I für $h$ und $S$ eingeführt werden. Wie das Verfahren III auf der Voraussetzung fusst, dass die zweite und die höheren Potenzen jener Differenz $h$, die zwischen dem aus $h$ und $S$ berechneten und dem aus dem beobachteten Verhältnisse $\frac{r}{n}$ direkt abgeleiteten Werthe von $(D-S) h$, bez. $(S-D) h$ besteht, ganz vernachlässigt werden können, so gründet sich auch das Verfahren I seinerseits auf die Voraussetzung, dass die zweite und die höheren Potenzen der plausibelsten Verbesserungen der eingeführten Näherungswerthe verschwindend klein seien. -

Im Bisherigen ist vorausgesetzt, dass allen beobachteten Verhältnissen $\frac{r}{n}$ eine und dieselbe Versuchszahl $n$ zu Grunde liege. Ist dies nicht der Fall und besitzen demgemäss die verschiedenen Werthe von $\frac{r}{n}$ auch an und für sich verschiedenes Gewicht, so hat man bei Anwendung des Verfahrens III jedem beobachteten $\frac{r}{n}$ oder vielmehr dem nach Gleichung (1), bez. (2), daraus folgenden $\tau$ das Gewicht $I \times G$ beizulegen, wo $I$ die obige Bedentung hat und $G$ das dem beobachteten $\frac{r}{n}$ in Folge der ihm zu Grunde liegenden Versuchszahl zukommende Gewicht bedeutet, und dann $h$ and $S$ in der obigen einfachen Weise aus den Werthen von $\tau$ abzuleiten. Da in den meisten der bisherigen Versuchsreihen die verschiedenen Werthe von $\frac{r}{n}$ wegen Verschiedenheit der ihnen zu Grunde liegenden Versuchszahlen $n$ nicht gleiche Genauigkeit besitzen, so würde man doch auch bei Anwendung des ungenaueren Verfahrens II sich oft genöthigt sehen, die verschiedenen Werthe von $z$ mit verschiedenen Gewichten $G$ in Rechnung zu bringen. Es erfordert daher das wesentlich genauere Verfahren III, nach welchem die in Anschlag zu bringenden Gewichte $=\Gamma \times G$ sind, in vielen Fällen gar nicht merklich mehr Mähe und Zeit als Verfahren II. --

Wie aus Gleichung (3) und (4) leicht zu erkennen, lassen sich dann, wenn ausser den richtigen und falschen Fällen auch unentschiedene Fälle vorkommen, die Werthe $h^{\prime}$ und $S^{\prime \prime}$ (vgl. S. 195 f.) aus den beobachteten Verhältnissen $\frac{f}{n}$ ganz ebenso nach Verfahren I, II und III berechnen, wie die Werthe $h$ und $S$ nach den vorstehenden $\$ \S$. aus den Verhältnissen $\frac{r}{n}$ zu bestimmen sind. Falls 
sich aus den Versuchen mit voller Sicherheit ergibt, dass $h=h^{\prime}$ ist, so wird es sich späterhin empfehlen, die 3 Unbekannten $h, S$ und $S^{t}$ aus den beobachteten Werthen von $\frac{r}{n}$ und $\frac{f}{n}$ gemeinschaftlich zu berechnen. Bezeichnen wir die aus $\frac{r_{1}}{n}, \frac{r_{2}}{n}$ u. s. f. abgeleiteten Werthe von $(D-S) h$ mit $x_{1}, t_{2}$ u. s. f. und die aus $\frac{f_{1}}{n}, \frac{f_{2}}{n}$ u. s. f. sich ergebenden Werthe von $\left(D-S^{\prime}\right) h^{\prime}$ mit $\tau_{1}^{\prime}, \tau_{2}^{\prime}$ u. s. f., so wird also dann, wenn sich $h^{\prime}=h$ herausstellt, das Verfahren III am besten in der Weise Anwendung finden; dass man die Gleichungen :

$$
\begin{gathered}
\left(D_{1}-S\right) h=\tau_{1} \\
\left(D_{2}-S\right) h=\tau_{2} \\
\left(D^{3}-S\right) h=\tau_{3} \\
\text { u. s. f. } \\
\left(D_{1}-S^{\prime}\right) h=\tau_{1}^{\prime} \\
\left(D_{2}-S^{\prime}\right) h=\tau_{2}^{\prime} \\
\left(D_{3}-S^{\prime}\right) h=\tau_{3}^{\prime}
\end{gathered}
$$

u. s. f.

aufstellt und dieselben, indem man $S h=k$ und $S^{\prime} h=k^{\prime}$ setzt, unter Bericksichtigung der Gewichte $\Gamma \times G$ sämmtlich gemeinsam zur Bestimmung der 3 Unbekannten $h, S$ und $S^{*}$ benutzt.

\section{s. 7.}

Da die Annahme, dass die Schwelle der richtigen Fälle eine unregelmässig schwankende sei und demgemäss $=S \pm \delta$ gesetzt werden mïsse, eine ganz erfahrungsmässige ist und die Behauptung, dass demnach ein richtiger Fall immer nur dann eintrete, wenn $D>S \pm \delta$ sei, eine ganz unanfechtbare ist, so scheinen die Verfahrungsweisen I und III und die denselben zu Grunde liegenden Fundamentalgleichungen (1) bis (4) nur in einer einzigen Hinsicht einem Bedenken unterworfen zu sein. Man kann nämlich zweifeln, ob für die zufälligen Fehlergrössen $\pm \delta$ wirklich das im Bisherigen schlechthin als gültig vorausgesetzte Gauss'sche Fehlergesetz bestehe. Zur näheren Begründung dieses Zweifels könnte man erstens bemerken, dass nach dem Gauss'schen Fehlergesetze die Grenze der möglichen Fehlergrössen gleich $\pm \infty$ sei und mithin nach diesem Gesetze bei allen möglichen Spitzenabständen $D$ 
noch falsche Fälle vorkommen müssten, insofern anch bei Anwendung sehr grosser $D$ 's immer noch Fälle eintreten müssten, wo der Fehler $\delta$ positiv und $>D-S$ ausfalle und demgemäss $D$ $<S \pm \delta$ sei. Es sei jedoch in den vorliegenden Versuchsreihen für zahlreiche nicht gerade sehr grosse Spitzenabstände das Verhältniss $\frac{r}{n}=1$ erhalten worden. Zweitens kann man geltend machen, wie sehr anch die Schwelle der richtigen Fälle eine zufällig schwankende sein möge, auf jeden Fall müsse, da ein negativer Werth dieser Schwelle keinen Sinn habe, $S \pm \delta>0$ sein; mithin könne der negative Fehler - $\delta$ seinem absoluten Werthe nach niemals $>S$, geschweige denn $=-\infty$ sein, und das Gauss'sche Fehlergesetz müsse daher für die Fehlergrössen $\pm \delta$ ungiiltig sein ${ }^{1}$ ).

Der erstere dieser beiden Einwände liesse sich vielleicht durch den Hinweis darauf beseitigen, dass die Anzahl der Versuche immer eine zu geringe gewesen sei, und dass bei ins Unendliche fortgesetzten Versuchen für jedes endliche $D$ das Verhältniss $\frac{r}{n}$ sich $<1$ herausstellen würde. Dem zweiten Einwande aber dürfte auch bei vertiefter Betrachtungsweise dieses Gegenstandes eine gewisse Berechtigung nicht abzustreiten sein. Nur ist Folgendes zu bemerken. Wenn auch das thatsächlich guiltige Wahrseheinlichkeitsgesetz der Fehler $\pm \delta$ streng genommen nicht mit dem Gauss'schen Gesetze übereinstimmt, weil das Maximum jener Fehler eine endliche Grösse ist, so kann ersteres Gesetz doch immerhin mit letzterem innerhalb weiter Grenzen in der Weise mehr oder weniger genau übereinstimmen, dass die Wahrscheinlichkeiten der. allermeisten und allerhäufigsten, nämlich derjenigen Fehlergrössen, welche nicht allzu nahe an das Fehlermaximum herankommen, thatsächlich fast ganz in denselben Verhältnissen zu einander stehen, in denen sie bei strenger Guiltigkeit des Gauss'schen Gesetzes zu einander stehen würden, und eine Abweichung vom Gauss'schen Gesetze sich erst dann merklich macht, wenn sich die Fehler-

1) Kürze halber ist im Obigen vorausgesetzt, dass nur richtige und falsche Fälle erhalten würden. Es lassen sich jedoch die obigen Betrachtungen leicht auf den Fall übertragen, wo auch noch unentschiedene Fälle vorkommen. Was nach Obigem von den Fehlern $\pm \delta$ gilt, gilt dann in entsprechender Weise auch noch von den Fehlern $\pm \delta^{\prime}$. 
grössen ihrem endlichen Maximalwerthe sehr bedeutend nähern. Da nun die Wahrscheinlichkeiten derjenigen Fehler $\pm \delta$, welche sehr nahe an ihren Maximalwerth herankommen, bei Benutzung der Gleichungen (1) und (2) und der darauf fussenden obigen Verfahrungsweisen im Allgemeinen gar nicht weiter in Betracht kommen, sondern es sich dabei immer nur um die Wahrscheinlichkeiten derjenigen Fälle handelt, in denen die Fehler $\pm \delta$ gewisse, von ihrem Maximalwerthe mehr oder weniger weit entfernte Grössen $(D-S$, bez. $S-D)$ nicht übersteigen, so können also immer noch jene Gleichungen innerhalb weiter Grenzen hinreichend gültig und die darauf fussenden Verfahrungsweisen I und III thatsächlich genügend sein. Man wird daher behufs Verwerthung der beobachteten Verhältnisse $\frac{r}{n}$ in diesem Gebiete stets am besten in der Weise verfahren, dass man zunächst mittelst Verfahrens III die Werthe von $h$ und $S$ bestimmt, und dann untersucht, ob die mit Hülfe dieser Bestimmungen nach Gleichung (1) und (2) rïckwärts wieder berechneten Werthe von $\frac{r}{n}$ mit den thatsächlich beobachteten Werthen dieses Verhältnisses hinlänglich übereinstimmen. Ist letzteres nicht der Fall, so ist die Voraussetzung, dass das Gauss'sche Gesetz für die Wahrscheinlichkeiten der Fehler $\pm \delta$ in der obigen Weise annähernd gültig sei, für unzutreffend zu erachten und man hat dann zu untersuchen, welches andere Fehlergesetz zu einer hinlänglich befriedigenden Uebereinstimmung der beobachteten und der rückwärts wieder berechneten. Werthe von $\frac{r}{n}$ führt. Diese letztere Untersuchung muss sich principiell stets durchführen lassen, dürfte aber in praxi meist eine ziemlich schwierige und langwierige sein, weil der Werth $S$, von welchẻm aus. die Fehler $\pm \delta$ zu rechnen sind, selbst von der Art des vorausgegesetzten und zu prüfenden Wahrscheinlichkeits'gesetzes jener Fehler abhängig ist. Fehlergesetze, die man nächst dem Gauss'schen Wahrscheinlichkeitsgesetze namentlich ins Auge zu fassen hat, sind z. B. folgende:

$$
\begin{aligned}
& w=\frac{2}{\alpha} \sin ^{2}\left(\frac{\pi}{2}\left(1-\frac{\delta}{\alpha}\right)\right), \\
& w=\frac{3}{2 \alpha}\left(1-\frac{\delta^{2}}{\alpha^{2}}\right) \text { u. dgl. m., }
\end{aligned}
$$


wo $w$ die Wahrscheinlichkeit des Fehlers $\pm \delta$ und $\alpha$ das endliche Fehlermaximum bedeutet. Mag sich nun die Annahme dieses oder jenes Fehlergesetzes als zutreffend erweisen, auf jeden Fall ist die Betrachtungsweise, von welcher wir in $\$$. $1 \mathrm{ff}$. ausgegangen sind, vollkommen triftig und unter allen Umständen geeignet, zu einer richtigen Verwerthung der beobachteten Verhältnisse $\frac{r}{n}$ zu führen. In Gleichnng (1) und (2) bleiben die Grenzen der Integration unter alleǹ Umständen ganz dieselben; ändern kann sich nur die Art des vorausgesetzten Wahrscheinlichkeitsgesetzes. Wie sich ferner auf Grund der Gleichungen (1) und (2) zwei Unbekannte berechnen lassen, die Werthe $S$ und $h$, von denen der letztere (da er $=\frac{1}{\delta_{m} \sqrt{\pi}}$ ist) uns über den mittleren Werth der Fehler $\pm \delta$ Auskunft gibt, so wird man auch dann, wenn sich ein anderes als das Gauss'sche Fehlergesetz als giiltig erweist, ausser dem Werthe $S$ noch eine andere Grösse berechnen können, welche entweder das Maximum oder der mittlere Werth der Fẹhler $\pm \delta$ oder eine davon abhängige Grösse ist und uns demgemäss gleichfalls über den Spielraum der (durch die Fehler $\pm \delta$ repräsentirten) zafälligen Schwankungen der Raumschwelle Aufschluss gibt. Sollte sich der schwieriger zu behandelnde Fall herausstellen, dass die Wahrscheinlichkeit des Fehlers $\pm \delta$ keine gerade Funktion der Fehlergrösse ist, sondern die Wahrscheinlichkeiten gleich grosser positiver und negativer Fehlergrössen versehieden siǹd, so wird man, indem man das eine Mal nur diejenigen Werthe von $\frac{r}{n}$, welche $>1 / 2$ sind, das andere Mal nur diejenigen, welche $<1 / 2$ sind, benutzt, aus den beobachteten Verhältnissen $\frac{r}{n}$ ausser dem Werthe $S$, für welchen $\frac{r}{n}=1 / 2$ ist, noch 2 andere Grössen ableiten können, von denen die eine über den Spielraum der positiven, die andere iiber den Spielraum der negativen Werthe des Fehlers $\delta$ Auskunft gibt. Eine nähere Erörterung aller dieser Eventualitäten würde uns za weit führen und dürfte ausserdem auch übérflüssig sein, da man sich aus den bisherigen und den folgenden Entwickelungen das richtige Verfahren für die verschiedenen denkbaren Eventualitäten leicht abnehmen kann. 
III. Empirische Prïfung der aufgestellten Fundamentalgleichungen und der daraf fussenden Berechnungsweisen.

§. 8.

Will man eine empirische Prüfung der Fundamentalgleichungen (1) bis (4) und der darauf fussenden obigen 3 Berechnungsweisen von $h$ und $S$ auf Grund der zahlreichen, von Vier ord t's Schülern ${ }^{2}$ ) angestellten Versuchsreihen unternehmen, so hat man nach jenen Verfahrungsweisen aus den Verhältnissen $\frac{r}{n}$ und $\frac{f}{n}$, die an einer Hautstelle für verschiedene Spitzenabstände erhalten wurden, die Werthe $h$ and $S, h^{6}$ und $S^{4}$ zu bestimmen und dann mittelst dieser Werthe auf Grund der Gleichungen (1) bis (4) rüickwärts wieder für jeden Spitzenabstand $D$ das żugehörige Verhältniss $\frac{r}{n}$ und $\frac{f}{n}$ zu berechnen. Ans dem Grade der Uebereinstimmung, in welcher die in dieser Weise berechneten und die thatsächlich beobachteten Werthe von $\frac{r}{n}$ und $\frac{f}{n}$ uu einander stehen, lässt sich dann die Zulänglichkeit des angewandten Berechnungsverfahrens beurtheilen, vorausgesetzt, dass den einzelnen beobachteten Verhältnissen $\frac{r}{n}$ und $\frac{f}{n}$ eine himreichend grosse Anzahl von Versuchen zu Grunde liegt und die Versuchsresultate den weiterhin (in §.12) näher zu besprechenden Kriterien einer normal ausgefallenen Versuchsreihe hinlänglich entsprechen.

1) Vgl. R. Kottenkamp und H. Ullrich in der Zeitschrift für Biologie, Bd. VI, S. 37 ff., A. Pa u Ius, ebendaselbst Bd. VII, S. 237 ff., A. Riecker, ebendaselbst Bd. IX, S. 95 ff. und Bd. X, S. $177 \mathrm{ff}$, G. Hartmann, ebendaselbst Bd. XI, S. $79 \mathrm{ff}$ Noch nicht näher veröffentlicht sind die Versuchsresultate von Camerer (vgl. Vierordt in Gerhardt's Handbuch der Kinderkrankheiten I, S. 198 f.). Dasselbe gilt wohl auch von den Versuchen Knöller's (vgl. Vi erordt, ebendaselbst und in seinem Grundriss der Physiologie des Menschen, S. 343 und 346). Auch Volkmann (Ber. der K. Sächs. Ges. d. W., Math.-Phys. Cl., 10. Bd., 1858, S. 47 ff.) hat einige Versuchsreihen nach der Methode der r. u. f. Fälle im Gebiete des Ortssinnes der Haut angestellt. 
Was diejenigen Beobachtungswerthe von $\frac{r}{n}$ und $\frac{f}{n}$ betrifft, die $=0$ oder $=1$ sind und denen demgemäss nach Gleichung (1) bis (4) unendlich grosse Werthe von $(D-S) h$, bez. $(S-D) h$ entsprechen, so können dieselben bei Benutzung der Verfahrungsweisen II und III zwar nicht mit zur Berechnung von $h$ und $S$, $h^{\prime}$ und $S^{\prime}$ verwandt, wohl aber bei Vergleich der beobachteten und der berechneten Werthe von $\frac{r}{n}$ und $\frac{f}{n}$ mit herangezogen werden. Es muss fïr jedes $D$, für welches $\frac{r}{n}\left(\right.$ bez. $\left.\frac{f}{n}\right)=0$ oder $=1$ beobachtet worden ist, das zugehörige berechnete $\frac{r}{n}\left(\right.$ bez. $\left.\frac{f}{n}\right)$ wenigstens annähernd $=0$, bez. $=1$ ausfallen. Falls sich herausstellt, dass das Wahrscheinlichkeitsgesetz der Fehler $\pm \delta$ und $\pm \delta^{\prime}$ nieht mit dem Gauss'schen, sondern mit einem anderen Fehlergesetze annähernd iibereinstimmt, nach welchem das Maximum jener Fehler einen endlichen Werth besitzt, so hat man allerdings die Werthe von $\frac{r}{n}$ und $\frac{f}{n}$, die $=0$ oder $=1$ sind, anch bei Berechnung der Werthe $S$ und $S^{\prime}$ und der beiden Maxima der Fehler $\pm \delta$ und $\pm \delta^{\prime}$ nothwendig mit zu benutzen. Will man übrigens bei Benutzung der Verfahrungsweisen II and III die $=0$ und die $=1$ erbaltenen Werthe von $\frac{r}{n}$ und $\frac{f}{n}$ durchaus mit zur Bestimmung von $h$ und $S, h^{\prime}$ und $S^{\prime}$ verwenden, so kann man sich.dadurch helfen; dass man statt 0 einen sehr wenig von 0 abweichenden Werth, etwa 0,001, und statt 1 einen sehr wenig von 1 verschiedenen Werth, etwa 0,999, als beobachteten Werth von $\frac{r}{n}$, bez. $\frac{f}{n}$ betrachtet. In folgenden Tabellen babe ich mich jedoch allgemein darauf beschränkt, die $=0$ und die $=1$ erhaltenen Werthevon $\frac{r}{n}$ und $\frac{f}{n}$ nur bei Vergleich von Beobachtung und Berechnung mit heranzuziehen.

Um zunächst für die Behauptung, dass die Verfahrungsweisen I und III wesentlich genauer seien als Verfahren II, eine empirische Bestätigung anzuführen, habe ich in Tabelle II die Verhältnisse $\frac{r}{n}$, welche in einer der besten Versuchsreihen von Riecker (vgl. 
Zeitschr. f. Biol. X, S. 192, Versuchsreihe III. 6, am hinteren Ende der linea semicircularis ossis bregmatis angestelt) erhalten wurden, mit denjenigen Verbältnissen $\frac{r}{m}$ zusammengestellt, welche sich aus den mittels Verfahrens I, II und III erhaltenen Werthen von $h$ und $S$ für dieselben Spitzenabstände $D$ riickwärts wieder berechneten.

Tabelle II.

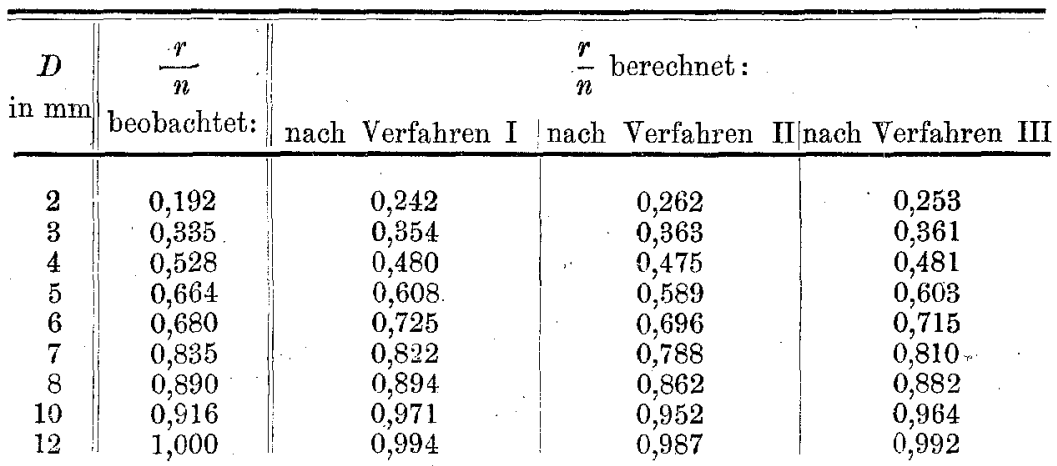

Obwohl in dieser Tabelle die nach Verfahren II berechneten Werthe von $\frac{r}{n}$, (abgesehen von dem für $D=8$ berechneten Werthe) von den beobachteten Werthen immer nach derselben Seite hin abweichen wie die nach Verfahren I und III berechneten Werthe, so stimmen doch die nach letzteren Verfahrungsweisen berechneten Werthe erheblich genauer mit den beobachteten Werthen ïberein. Denn während die Summe der Quadrate der Differenzen zwischen Beobachtung and Berechnung nach Verfahren II gleich 0,018832 ist, betrïgt dieselbe nach Verfahren III nur 0,014609 and nach Verfahren I nur 0,013585. Dem entsprechend stimmen auch die nach Verfahren III berechneten Werthe von $h$ and $S$ erheblich mehr mit den nach Verfahren I bestimmten Werthen iiberein als. diejenigen Werthe, welche das Verfabren II ergibt. Denn es findet $\operatorname{sich}^{1}$ )

1) Bei Bestimmung von $h$ und $S$ sind die Beobachtungswerthe $\frac{r}{n}$ sämmtlich als gleich genau betrachtet worden, da die ihnen zu Grunde liegenden Versuchszahlen $n$ nur wenig differiren. Dasselbe gilt von den in Tabelle III angeführten Beobachtungswerthen $\frac{r}{n}$ und $\frac{f}{n}$. - Die Näherungswerthe, welche bei obiger Anwendung des Verfahrens I für $h$ und $S$ eingeführt wurden, waren $h_{0}=0,204$ und $S_{0}=4,22$. 


$$
\begin{array}{cccc}
\text { nach Verfahren I: } h=0,2294 & S=4,1586 \\
" \quad & \quad \text { II: } h=0,2035 \quad & S=4,2167 \\
" & \quad \text { III }: h=0,2178 & S=4,1544
\end{array}
$$

'Wenn wir oben auf Grund theoretischer Erwägungen behauptet haben, der Mangel des Verfahrens II laufe im Grunde darauf hinaus, dass bei gleicher Genauigkeit aller beobachteten Verhältnisse $\frac{r}{n}$ dennoch diesen Verhältnissen verschiedene Gewichte beigelegt würden und zwar jedem Werthe von $\frac{r}{n}$ ein um so höheres Gewicht zugeschrieben werde, je weiter er nach unten oder oben hin von $1 / 2$ abweiche, so findet diese Behauptung in der nachweislich grösseren Genauigkeit des Verfahrens mit Gewichtscorrektionen ihre erfahrungsmässige Bestätigung. Die Richtigkeit jener Behauptung geht aber auch daraus hervor, dass, werin man aus den nach Verfahren II erhaltenen Werthen von $h$ und $S$, bez. $h^{\prime}$ und $S^{\prime}$, für die verschiedenen benutzten Spitzenábstände die Verhältnisse $\frac{r}{n}$, bez. $\frac{f}{n}$, rückwärts wieder berechnet, alsdann diejenigen der berechneten Verhältnisse $\frac{r}{n}$, bez. $\frac{f}{n}$, welche am weitesten von dem Werthe 1/2 abstehen, durchschnittlich am meisten mit ihren entsprechenden Beobachtungswerthen übereinstimmen. Dies muss sich namentlich bei denjenigen Versuchsreihen zeigen, bei denen mehrere sehr hohe, der Einheit nahekommende oder mehrere sehr niedere, von 0 wenig verschiedene Werthe von $\frac{r}{n}$ oder $\frac{f}{n}$ erhalten wurden, so z. B. bei der von Kottenkamp und Ullrich an der Volarseite des Oberarms (Lokalität b, vgl. Zeitschr. f. Biol. VI, S. 44) angestellten Versuchsreihe, auf welche sich folgende Zu-

\begin{tabular}{|c|c|c|c|c|c|c|c|}
\hline Beobachtet: & 0,50 & 0,73 & 0,80 & 0,90 & 0,94 & 0,97 & 0,99 \\
\hline Berechnet & & & & & & & \\
\hline $\begin{array}{l}\text { Verfahren II: } \\
\text { Berechnet }\end{array}$ & 0,615 & 0,692 & 0,761 & 0,87 & 0,938 & 0,975 & 0,991 \\
\hline Verfahren III & 0,563 & 0,669 & 0,762 & 0,897 & $0,966^{\circ}$ & 0,991 & 998 \\
\hline
\end{tabular}
sammenstellung bezieht.

$$
\text { Werthe von } \frac{r}{n} \text {. }
$$


Wie man sieht, stimmen mit den 3 höchsten Beobachtungswerthen von $\frac{r}{n}$ die nach Verfahren II für dieselben $D$ 's berechneten Werthe dieses Verhältnisses bedeutend mẹr überein als die nach Verfahren III berechneten Werthe; was eben eine nothwendige Folge davon ist, dass das Verfahren II diesen 3 höchsten Beobachtungswerthen viel zu hohe falsche Gewichte beilegt. Die Summe der Quadrate der Differenzen zwischen Beobachtung und Berechnung ist aber doch für das Verfahren II eine ganz erheblich grössere $(=0,017120)$ als für das Verfahren III $(=0,010324)$. Die Werthe von $h$ und $S$, welche ersteres Verfahren ergibt, weichen von den nach letzterem Verfahren erhaltenen Werthen ganz beträchtlich ab. Denn es findet sich nach Verfahren II $h=0,147$ und $S=14,595$, hingegen nach Verfahren III $h=0,196$ und $S=15,432$. Man hat daher stets das Verfahren III, welches das genauere ist und thatsächlich auch einen regelmässigeren Gang von $h$ und $S, h^{*}$ und $S^{\prime}$ ergibt, dem Verfahren II vorzuziehen und letzteres im Grunde durchaus fehlerhafte Verfahren tiberhaupt ganz zu vermeiden.

'Um noch einen weiteren Einblick' zul gewähren, inwieweit sich das Verfahren mit Gewichtscorrektionen an den bisherigen Versuchsreihen bewährt, gebe ich in der folgenden Tabelle III noch eine Zusammenstellung der in einigen der bisherigen Versuchsreihen erhaltenen Werthe von $\frac{r}{n}$ und der entsprechenden nach Verfahren III berechneten Werthe dieses Verhältnisses. Die Versuchsreihen dieser Tabelle sind nicht beliebig, sondern deshalb ausgewählt worden, weil sie von den zur Zeit vorliegenden, mehr als 4 Beobachtungswerthe von $\frac{r}{n}$ ergebenden Versuchsreihen die einzigen sind, deren Resultate den in $\$ .12 \mathrm{zu}$ besprechenden, auf der Voraussetzung der Zulänglichkeit des Verfahrens III nicht fussenden, ganz objektiven Kriterien einer ganz normal ausgefallenen Versuchsreihe durchaus entsprechen. Versuchsreihe I, II, III und IV sind von Kottenkamp und Ullrich in der Metacarpalregion der Hand (Volarseite, Grenze des 4. und 3. Viertels) und am Oberarm (Volarseite, Lokalität a, und Dorsalseite, Lokalität a und c) angestellt. Versuchsreihe $V$, bei welcher anch, unentschiedene Fälle vorkamen und daher die beobachteten und berech- 
neten Werthe von $\frac{f}{n}$ mit besonders aufgefuihrt werden mussten, ist die von Riecker am rothen Theile der Oberlippe angestellte Versuchsreihe. Bei jeder Versuchsreihe sind zugleich die nach Verfahren III gefundenen Werthe von $h$ und $S$, bez. $h^{\prime}$ und $S^{\prime}$, mit angegeben.

Tabelle III.

\begin{tabular}{|c|c|c|c|c|c|c|c|c|c|c|c|}
\hline \multirow{2}{*}{\multicolumn{2}{|c|}{$\begin{array}{l}\text { Versuchs- } \\
\text { reihe 1. } \\
h=0,427 \\
S=1,939\end{array}$}} & \multicolumn{2}{|c|}{$\begin{array}{l}\text { Versuchs- } \\
\text { reihe II. }\end{array}$} & \multicolumn{2}{|c|}{$\begin{array}{l}\text { Versuchs- } \\
\text { reihe III. }\end{array}$} & \multicolumn{2}{|c|}{$\begin{array}{l}\text { Versuchs- } \\
\text { reihe IV. }\end{array}$} & \multicolumn{4}{|c|}{ Versuchsreihe $\nabla$. } \\
\hline & & \multicolumn{2}{|c|}{$\begin{array}{l}h=0,239 \\
S=14,956\end{array}$} & \multicolumn{2}{|c|}{$\begin{array}{l}h=0,276 \\
S=15,097\end{array}$} & \multicolumn{2}{|c|}{$\begin{array}{l}h=0,304 \\
S=17,670\end{array}$} & \multicolumn{2}{|c|}{$\begin{array}{l}h=2,192 \\
S=1,055\end{array}$} & \multicolumn{2}{|c|}{$\begin{array}{l}h^{\prime}=2,029 \\
S^{\prime \prime}=1,025\end{array}$} \\
\hline \multicolumn{2}{|c|}{$r$} & \multicolumn{2}{|c|}{$\boldsymbol{r}$} & \multicolumn{2}{|r|}{$r$} & \multicolumn{2}{|c|}{$\boldsymbol{r}^{*}$} & \multicolumn{2}{|c|}{$r$} & \multicolumn{2}{|c|}{$f$} \\
\hline \multirow{2}{*}{$\begin{array}{l}\text { beob- } \\
\text { achtet: }\end{array}$} & & \multicolumn{2}{|c|}{$n$} & \multicolumn{2}{|c|}{$n$} & \multicolumn{2}{|c|}{$n$} & \multicolumn{2}{|c|}{-1} & \multicolumn{2}{|c|}{-} \\
\hline & $\begin{array}{l}\text { perech- } \\
\text { net: }\end{array}$ & $\begin{array}{c}\text { beob- } \\
\text { achtet: } \\
0.50\end{array}$ & $\begin{array}{c}\text { berech- } \\
\text { net: } \\
0.506\end{array}$ & beob- & $\begin{array}{l}\text { berech- } \\
\text { net: }\end{array}$ & beob- & berech- & $\begin{array}{l}\text { beob- } \\
\text { achtet: }\end{array}$ & $\begin{array}{l}\text { berech } \\
\text { net: }\end{array}$ & $\begin{array}{l}\text { beob- } \\
\text { achtet: }\end{array}$ & $\begin{array}{l}\text { berech- } \\
\text { net: }\end{array}$ \\
\hline 0,51 & 0,515 & 0,64 & 0,638 & 0,62 & 0,637 & 0,56 & 0,556 & 0 & 0,001 & 1,000 & 0,998 \\
\hline 0,75 & 0,739 & 0,76 & 0,756 & 0,80 & 0,771 & 0,74 & 0,716 & 0,043 & 0,043 & 0,931 & 0,934 \\
\hline 0,89 & 0,894 & 0,86 & 0,849 & 0,88 & 0,871 & 0,85 & 0,842 & 0,432 & 0,432 & 0,531 & 0,529 \\
\hline 0,96 & 0,968 & 0.94 & 0,956 & 0,95 & 0,972 & 0,94 & 0.969 & 0,916 & 0,916 & 0,084 & 0,087 \\
\hline 1,00 & 0,993 & 1,00 & 0,991 & 1,00 & 0,996 & 1,00 & 0,997 & 1,000 & 0,998 & 0 & 0,003 \\
\hline
\end{tabular}

Die Uebereinstimmung zwischen den beobachteten und berechneten Werthen dieser Tabelle ist ohne Zweifel als eine befriedigende zu bezeichnen. Entsprechende Resultate erhält man auch bei Benutzung anderer, nicht ganz normal ausgefallener Versuchsreihen, falls nur die in Folge der zu geringen Versuchszahl erhaltenen Unregelmässigkeiten des Ganges der beobachteten Verhältnisse $\frac{r}{n}$ und $\frac{f}{n}$ nicht zu augenfällige sind. Wir können daher ohne Bedenken behaupten, dass sich die Fundamentalgleichungen (1) bis (4) und das darauf fussende Verfahren III an den zur Zeit vorliegenden Versuchsreihen hinlänglich bewähren.

IV. Von den Vexirversuchen und der Mitverwendung ihrer Resultate.

§. 9.

Um die nöthige Voraussetzungslosigkeit bei der Versuchsperson herbeizuführen, wurden bei sämmtlichen bisherigen Versuchsreihen (abgesehen von denen Volkmann's) sogenannte Vexirversuche mit angestellt, $d . h$. es wurde "in buntem Wechsel mit 
den Hauptversuchen, ohne dass die Versuchsperson etwas merken konnte", nur ein Punkt der Haut berïhrt. Bei diesen Vexirversuchen wurde nun merkwürdiger Weise nicht immer das Urtheil gefällt, es sei nur ein Hautpunkt beriihrt worden, sondern es entstand trotz der thatsächlich einfachen Berihrung zuweilen der Eindruck einer Doppelberiihrung. Erwägen wir nun, dass ein Eindruck der letzteren Art nach §. 2 nur immer dann eintreten kann, wenn der Spitzenabstand $D$ grösser als die Schwelle der richtigen Fälle, d. h. $D>S \pm \delta$ ist, so scheint, da bei den Vexirversuchen $D=0$ ist, die Thatsache der bei diesen Versuchen gefällten falschen Urtheile zu ergeben, dass die Schwelle der richtigen Fälle zuweilen $<0$ ist und negative Werthe annimmt, indem die negativen Werthe des Fehlers $\delta$ (ihren absoluten Grössen nach) $>S$ ausfallen. Andererseits aber muss uns, wie schon auf S. 208 bemerkt, das Vorkommen negativer Werthe der Schwelle der richtigen Fälle völlig undenkbar und sinnlos erscheinen. Diese Schwierigkeit löst sich leicht auf, wenn wir bedenken, dass die Wirkungen jeder Berïhrung eines Hauptpunktes sich in Folge einer äusseren, peripherischen und einer innern, im Nervensysteme vor sich gehenden Irradiation ${ }^{1}$ ) über einen grösseren, mehr oder weniger einem Kreise ähnlichen Hautbezirk erstrecken. Ist der grösste Abstand zweier in Folge dieser Irradiation noch hinlänglich erregter Punkte des Irradiationskreises $=d$, so verhält es sich also bei Berithrung nur eines einzigen Hautpunktes thatsächlich so, als würde bei mangelnder Irradiation der Berührungswirkungen die betreffende Hautgegend an 2 verschiedenen, um $d$ von einander abstehenden Punkten berührt. Die bei den Vexirversuchen gefällten falschen Entscheidungen weisen also thatsächlich nicht darauf hin, dass die Schwelle der richtigen Fälle allen Raisonnements zu Trotz zuweilen negativ sein kann,-sondern ergeben nur, dass diese Schwelle zuweilen kleiner als jener Abstand $d$ sein kann. Bezeichnen wir den Mittelwerth der Schwelle der richtigen Fälle (ans weiterhin leicht erkennbarem Grunde) statt wie bisher mit $S$ vielmehr mit $s$ und die Zahl der Fälle, in denen bei den Vexirversuchen, deren Gesammtzahl $=n$ sei, der Eindruck einer Doppelberührung entsteht, mit $r_{0}$, so ist nach Vorstehendem

1) Vgl. J. Bernstein, Untersuchungen äber den Erregungsvorgang im Nerven- und Muskelsystem, S. $170 \mathrm{f}$. und $181 \mathrm{ff}$. 
das Verhältniss $\frac{r_{0}}{n}$ gleich der Wahrscheinlichkeit der Fälle, in denen die Schwelle der richtigen Fälle (d. i. $s \pm \delta)<d$, mithin $\delta<d-s$ ist. Da $\frac{r_{0}}{n}$ der Erfahrung gemäss stets $<1 / 2$ ausfällt, mithin, wie leicht zu erkennen, $d$ stets $<s$ ist, so kann also bei den Vexirversuchen der Eindruck einer Doppelberiihrung nur dann entstehen, wenn die Fehlergrösse $\delta$ negativ und ihrem absoluten Werthe nach $>s-d$ ist. Folglich ist unter der Voraussetzung, dass für die Fehler $\pm \delta$ das Gauss'sche Wabrscheinlichkeitsgesetz mit binlänglicher Annäherung guiltig sei, allgemein

$$
\frac{r_{0}}{n}=\frac{1}{2}-\frac{1}{V \pi} \int_{o}^{(s-l) h} e^{-t^{2} d t} .
$$

Werden bei den Haupt- und Vexirversuchen ausser den richtigen und falschen Fällen auch noch unentschiedene Fälle erhalten, so gilt für die Fälle, wo die einfache Berührung den Eindruck einer Doppelberïhrung hervorruft, offenbar immer noch diese Formel (7). Ausserdem aber gilt, wie sich nach Vorstehendem leicht ableiten lässt, für die relative Anzahl $\frac{f_{0}}{n}$ derjenigen Fälle, in denen bei den Vexirversuchen der Eindruck einer einfachen Beriihrung entsteht, die Gleichung:

$$
\frac{f_{0}}{n}=\frac{1}{2}+\frac{1}{V_{\pi}^{-}} \int_{a}^{\left(s^{\prime}-d\right) h^{t}} e^{-t^{2}} d t,
$$

wo $s^{\prime}$ den Mittelwerth der Sehwelle der unentschiedenen Fälle und $h^{\prime}$ das Präcisionsmaass dieser Schwelle bedeutet.

§. 10 .

Jene Irradiation der Berührungswirkungen muss sich offenbar ebenso wie bei den Vexirversuchen auch bei den Hauptversuchen geltend machen, wo thatsächlich eine Doppelberïhrung stattfindet.

$$
\begin{aligned}
& A^{4} \quad A \quad B \quad B^{\prime} \\
& \hline
\end{aligned}
$$

Es möge $A$ den einen und $B$ den andern der beiden Berïhrungspunkte bedeuten, also der Spitzenabstand $D$ gleich $A B$ sein; ferner sei $B^{\prime}$ der von $A$ am weitesten entfernte noch hinlänglich erregte Hautpunkt des um $B$ sich erstreckenden Irradiationskreises und 
$A^{\prime}$ sei der von $B$ und $B^{\prime}$ am weitesten entfernte noch hinlänglich erregte Hautpunkt des andern, um $A$ sich erstreckenden Irradiationskreises. Alsdann ist die für unsere Auffassung thatsäehlich in Betracht kommende Distanz nicht $=A B=D$, sondern vielmehr $=A^{\prime} B^{\prime}=D+d$, wo $d$ wie oben den weitesten Abstand zweier noch hinlänglich erregter Punkte des Irradiationskreises bedeutet, mithin $\frac{d}{2}=A A^{\prime}=B B^{\prime}$ ist, und wo der Werth von $d$ mit der Lokalität der untersuchten Hautstelle sich ändern kann. Will man also genau sein, so hat man in den Fundamentalgleichungen (1) bis (4) (vgl. S. 193 und S. 196) iuberall $D+d$ zu setzen, wo bisher blos $D$ stand, mithin in jenen Gleichungen da, wo bisher $D-S$, bez. $D-S^{\prime \prime}$, stand, vielmehr $D-(s-d)$, bez. $D-\left(s^{\prime}-d\right)$, und, wo $S-D$, bez. $S^{\prime \prime}-D$ stand, vielmehr $(s-d)-D$, bez. $\left(s^{\prime}-d\right)-D$ zu setzen. Jene Fundamentalgleichungen behalten also nach wie vor ihre Gültigkeit; nur hat man streng genommen den darin vorkommenden Werth $S$, bez. $S^{\iota}$, gleich $s-d$, bez. gleich $s^{\prime}-d$, zu setzen, d. h. unter dem mit $S$, bez. $S^{\prime}$, bezeichneten Werthe nicht den wirklichen Mittelwerth der Schwelle der richtigen, bez. unentschiedenen Fälle, sondern vielmehr die Differenz zwischen diesem Mittelwerthe und dem grössten Abstande zweier hinlänglich erregter Hautpunkte des Irradiationskreises zu verstehen. Gibt man dem Werthe $S$, bez. $S^{\prime}$, diese Bedeutung, so ergeben sich, wenn man $D=0$ und demgemäss $\frac{r}{n}=\frac{r_{0}}{n}$ und $\frac{f}{n}=\frac{f_{0}}{n}$ setzt, ans Gleichung (2) und (3) die obigen Gleichungen (7) und (8).

Aus Vorstehendem ergibt sich, dass die Fälle, wo bei den Vexirversuchen der Eindruck einer Doppelberührung entsteht, von denjenigen Fällen, wo ein solcher Eindruck bei wirklicher Berührung durch 2 nur wenig von einander entfernte. Spitzen entsteht, gar nicht wesentlich verschieden sind, und dass es keineswegs sachgemäss sein dürfte, wenn Paulus (a. a. 0. S. 241) nach den Resultaten der Vexirversuche ,die Zuverlässigkeit der Entscheidungen des Versuchsindividuums" beurtheilt wissen will. Man erkennt aus dem Vorstehenden leicht, in welcher Weise man bei einer möglichst vollständigen Verwerthung der erhaltenen Ver. suchsresultate die Ergebnisse der Vexirversuche mit zu verwenden hat. Setzt man das Produkt $(s-d) h$ einfach $=S h=k$ (vgl. §. 5) und ebenso das Produkt $\left(s^{*}-d\right) h^{\prime}$ einfach $=S^{\prime} h^{\prime}=k^{\prime}$ und be- 
zeichnet man den wach Gleichung (7), bez. (8), aus den Resultaten der Vexirversuche abgeleiteten Werth von $-k$, bez. $-k^{\prime}$, mit $x_{0}$, bez. $\boldsymbol{u}_{0}^{\prime}$, so hat man bei Anwendung des Verfahrens III die Gleichungen

$$
\begin{gathered}
-k=\tau_{0} \\
D_{1} h-k=\tau_{1} \\
D_{2} h-k=\tau_{2} \\
\text { u. s. f. } \\
-k^{\prime}=\tau_{0}^{\prime} \\
D_{1} h^{\prime}-k^{\prime}=\tau^{\prime}{ }_{1} \\
D_{2} h^{\prime}-k^{\prime}=\tau_{2}^{\prime} \\
\text { u. s. f. }
\end{gathered}
$$

aufzustellen und dann auf Grund dieser Gleichungen unter Berücksichtigung der Gewichte $G \times I$ die Werthe von $h$ und $k, h^{\prime}$ und $k^{\prime}$ zu berechnen. -

Die Triftigkeit der im Vorstehenden angegebenen Mitverwendung der bei den Vexirversuchen erhaltenen Resultate lässt sich auf Grund der vorliegenden Versuchsreihen anch empirisch nachweisen. Vergleicht man die beobachteten Werthe von $\frac{r_{0}}{n}$ und $\frac{f_{0}}{n}$ mit denjenigen Werthen dieser Verhältnisse, die sich nach Gleichung (7) und (8) aus den nach vorstehendem (die Vexirversuche mit berücksichtigenden) Verfahren erhaltenen Werthen von $h$ und $S, h^{\prime}$ und $S^{\prime}$ berechnen, so stimmen die Beobachtungswerthe mit den Berechnungswerthen im Allgemeinen nicht weniger überein als die zu irgend einem endlichen Spitzenabstande zugehörigen Beobachtungswerthe von $\frac{r}{n}$ und $\frac{f}{n}$ mit ihren entsprechenden Berechnungswerthen übereinstimmen. Folgende Zusammenstellung enthält die Verhältnisse $\frac{r_{0}}{n}$ und $\frac{f_{0}}{n}$, die an den 6 oberen der von Riecker untersuchten Stellen des Unterschenkels von diesem Beobachter erhalten wurden, nebst den entsprechenden Berechnungswerthen.

$$
\begin{array}{cccccccc}
\frac{r_{0}}{n} \text { beobachtet: } & 0,106 & 0,241 & 0,122 & 0,120 & 0,102 & 0,198 \\
& \text { berechnet: } & 0,082 & 0,245 & 0,101 & 0,140 & 0,069 & 0,226 \\
\frac{f_{0}}{n} \text { beobachtet: } & 0,856 & 0,725 & 0,833 & 0,850 & 0,843 & 0,765 \\
& \text { berechnet: } & 0,871 & 0,717 & 0,863 & 0,847 & 0,893 & 0,696
\end{array}
$$


Die Werthe von $\frac{r_{0}}{n}$, welche von Riecker an der 7. und 8. der von ihm untersuchten Hattstellen des Unterschenkels erhalten wurden, sind ganz unzweifelhaft zu gering ausgefallen und daher nebst den entsprechenden Werthen von $\frac{f_{0}}{n}$ in der vorstehenden $\mathrm{Za}$ sammenstellung nicht mit berïeksichtigt ${ }^{1}$ ). -

Es ist wichtig. im Ange zu behalten, dass die obigen Bemerkungen iber die Mitverwendung der Resultate der Vexirversuche nur unter der Voraussetzung Gültigkeit beanspruchen, dass während jeder Versuchsreihe mit den verschiedenen Spitzenabständen $D .(D=0$ eingeschlossen $)$ in buntem Wechsel operirt wurde. Sämmtliche Versuchsreihen von Paulus, Kottenkamp und Ullrich, Riecker und Hartmann sind in dieser Weise angestellt worden. Wird, abgesehen von den dazwischen fallenden Vexirversuchen, jedes $D$ eine ganze Reihe von Versuchen hindurch ganz allein zur Anwendung gebracht, so können leicht constante Einflüsse entstehen, welche die dem Obigen zu Grunde liegende Betrachtungsweise als unzulänglich erscheinen lassen und eine einheitliche Verwendung sämmtlicher Versuchsresultate behufs möglichst genauer Erforschang des Ortssinnes bedeutend erschweren.

V. Von der geeigneten Anstellung derVersuche und den Kriterien einer guten Versuchsreihe.

§. 11.

Wie man aus dem Bisherigen leicht erkennt, ist eine genaue Verwerthung der gewonnenen Zahlen richtiger und falscher Fälle nur dann mit Schwierigkeiten verbunden, wenn die Voraussetzung nicht erfüllt ist, dass für die Fehlergrössen $\pm \delta$ und $\pm \delta^{\prime}$ das

1) Obige Auffassung der Vexirversuche bedarf in gewisser Hinsicht noch näherer Erörterung. Indessen kommt es hier nicht darauf an, Auffassungen zu entwickeln, die einer Theorie des Ortssinnes gar keine Frage mehr übrig lassen, sondern nur darauf, Vorstellungen zu gewinnen, die zu einer vollständigen und sachgemässen Verwerthung der Versuchsresultate führen and sich an letzteren bewähren. Wie auf S. 283 näher bemerkt wird, erklärt sich durch obige Auffassung auch die merkwürdige Thatsache, dass $\frac{r_{0}}{n}$ in der Regel um so grösser ausfällt, je feiner der Ortssinn ist: 
Gauss'sche Wahrscheinlichkeitsgesetz mit hinlänglicher Annäherung guiltig: sei, und demgemäss das auf dieser Voraussetzung fussendé Verfahren III nicht als hinreichend triftig betrachtet werden darf. Wie es scheint, kann man jedoch mit ziemlicher Sicherheit auf das Erfülltsein dieser Voraussetzung rechnen, wenn man erstens, um etwaige constante Einflüsse auszuschliessen, die verschiedenen Spitzenabstände $D$ in buntem Wechsel zur Anwendung: gebracht hat, und wenn man zweitens die Verhältnisse $\frac{r}{n}$ und $\frac{f}{n}$, die zur Berechnung von $h$ and $S, h^{\star}$ und $S^{\prime}$ dienen sollen, nicht dadurch erhalten hat, dass man die Resultate verschiedener Versuchsreihen, denen verschiedene Werthe von $h$ und $h^{t}, S$ und $S^{\prime}$ entsprechen, zusammenlegte. Findet eine Zusammenlegung der Resultate derartiger verschiedener Versuchsreihen statt, so kann, wie sich leicht näher nachweisen lässt, das eigentlich gïltige Wahrscheinlichkeitsgesetz der Fehler $\pm \delta$ und $\pm \delta^{\prime}$, welches innerhalb gewisser Grenzen doch wohl mehr oder weniger mit dem Gauss'schen Gesetze übereinstimmen dürfte, nicht mehr rein zu Tage treten. Werden also Resultate, die an verschiedenen Hautgegenden von nicht ganz gleichem Ortssinne und Präcisionsmaasse, werden Resultate verschiedener Beobachter, werden endlich Resultate zusammengelegt, von denen die einen vor, die anderen nach vollendeter Uebung erhalten wurden, so ist durchaus nicht mehr darauf zu rechnen, dass das Wahrscheinlichkeitsgesetz der Fehler $\pm \delta$ und $\pm \delta^{\prime}$ mit dem Gauss'schen Gesetze annähernd übereinstimme und die Formeln (1) bis (4) und das darauf fussende Verfahren III sich als ganz geniigend erweisen. Was den Einfluss der Uebung: betrifft, so ist wohl zu berïcksichtigen, dass sich der Einfluss der Uebung des Ortssinnes einer bestimmten Hautstelle nach Volkmann's Untersuchungen (a. a. 0. S. 63 ff.) zwar anf die symmetrisch gelegene Hautstelle und in geringerem Maasse auch auf die benachbarten Hautstellen mit zu erstrecken scheint, nicht aber auch auf die übrigen Hautstellen. Es ist daher dann, wenn man genau untersuchen will, wie sich verschiedene Hautgegenden betreffs der Feinheit des Ortssinnes zu einander verhalten, unbedingt nothwendig, an jeder der zu untersuchenden Hautstellen diejenige Anzahl von Vorversuchen anzustellen, die erforderlich ist, um einen annähernd constant bleibenden, durch weitere Uebung nicht leicht erhöhbaren Werth der Ortsempfindlichkeit und des Präcisions- 
maasses zu erreichen. Ansser dem Einflusse der Uebung ist endlich auch noch die Hautrund ng wohl zu bericksichtigen. Schon Paulus (a. a. O. S. 244) hat bemerkt, dass der Sinn offenbar nicht den geradlinigen Abstand zweier Hautpunktè, sondern den wirklichen Abstand derselben auf der Hautoberfläche messe, und dass in Folge dessen die grösseren Spitzenabstände eine der Rundung der betreffenden Hautgegend entsprechende Correktur zu erfahren hätten. Es fragt sich in der That, $a b$ wir nicht noch eine grössere Uebereinstimmung zwischen den beobachteten und den aus $h$ and $S, h^{\prime}$ und $S^{\prime}$ rückwärts wieder berechneten Werthen von $\frac{r}{n}$ und $\frac{f}{n}$ erzielen würden, wenn die von den bisherigen Beobachtern benutzten Spitzenabstände $D$ einer Correktur wegen der Hantrundung unterworfen worden wären. Paulus hat allerdings die von ihm benutzten $D$ 's einer solchen Correktur unterworfen; seine Versuche sind jedoch zu wenig zahlreich, um eine langwierige hierauf bezügliche Untersuchung zu lohnen und eine wirklich entscheidende Prüfung dieser Frage zu verstatten.

\section{§. 12.}

Bei Ausfïhrung und Verwendung von Versuchsreihen, die nach der Methode der richtigen und falschen Fälle angestellt werden, darf man nie aus dem Auge verlieren, dass alle Begeln und Formeln, die für die Verwendung der Resultate soleher Versuchsreihen aufgestellt werden, stets von der Voraussetzung ausgehen müssen, dass die experimentell erhaltenen Werthe von $\frac{r}{n}$ und $\frac{f}{n}$ mit denjenigen Werthen, welche dem Wahrscheinlichkeitsgesetze der zufälligen Schwankungen der Ortsempfindlichkeit gemäss für die betreffenden Spitzenabstände die wahrscheinlichsten Werthe jener Verhältnisse sind, wenigstens annähernd übereinstimmen. Diese Voraussetzung muss dem Gesetze der grossen Zahlen gemäss im Allgemeinen sich um so mehr bestätigen und um so mehr erlaubt sein, je grösser die Zahl der angestellten Versuche ist; aber man kann auch bei grosser Versuchszahl nie absolut sicher sein, dass nicht einmal auch einer der sehr nnwahrscheinlichen Werthe für $\frac{r}{n}$ oder $\frac{f}{n}$ erhalten werde. Man darf daher nicht sofort auf einen unbemerkt gebliebenen Mangel der Versuchsweise, störende Einflüsse u. dergl. schliessen, wenn sich einmal in einer Versuchs- 
reihe eine auffallende Unregelmässigkeit und Abweichung von dem erwarteten Gange der Verhältnisse $\frac{r}{n}$ und $\frac{f}{n}$ herausstellt. Denn ein derartiges Resultat kann seinen Grund auch lediglich darin haben, dass die zufälligen Schwankungen der Schwelle der richtigen, bez. unentschiedenen, Fälle ein Verhalten gezeigt haben, das zwar als äusserst unwahrscheinlich, aber doch nicht als unmöglich zu betrachten war.

Da die Zahl der Versuche, die an einer bestimmten Hantstelle bei einem und demselben Spitzenabstande angestellt werden, immer nur eine beschränkte sein wird und namentlich die Versuchszahlen der bisherigen Versuchsreihen nur geringe sind, so ist es nothwendig, sich über die Kriterien klar zu werden, an denen man erkennen kann, ob die Resultate einer Versuchsreihe wenigstens annähernd mit dem wahrscheinlichsten Gange der Verhältnisse $\frac{r}{n}$ und $\frac{f}{n}$ übereinstimmen. Denn eben nur insoweit, als sie diesen Gang wenigstens annähernd befolgen, können die Ergebnisse einer Versuchsreihe zur Bestimmung der Feinheit des Ortssinnes, des Präcisionsmaasses $\mathfrak{u}$. dergl, dienen. Bei Bestimmung des wahrscheinlichsten oder des normalen Ganges der Verhältnisse $\frac{r}{n}$ und $\frac{f}{n}$ gehe ich lediglich von der Voraussetzung aus, dass die Wahrscheinlichkeit des Fehlers $\pm \delta$, bez. $\pm \delta^{\prime}$, bei wachsender Fehlergrösse auf keinen Fall zunehme, sondern entweder abnehme oder constant bleibe. Ob also das Wahrscheinlichkeitsgesetz jenes Fehlers, wie in den Fundamentalgleichungen (1) bis (4) vorausgesetzt, mit dem Gauss'schen Fehlergesetze iibereinstimme oder nicht, ob überhaupt die Wahrscheinlichkeit jenes Fehlers eine gerade Funktion der Fehlergrösse sei oder nicht, u. dergl. m. bleibt hier gänzlich dahingestellt. Aus jener durchaus statthaften Voraussetzung ergibt sich nun, wie leicht näher abzuleiten, betreffs des normalen Ganges der (auf eine und dieselbe Hautstelle bezüglichen) Verhältnisse $\frac{r}{n}$ und $\frac{f}{n}$ Folgendes: wächst der Spitzenabstand $D$, der zunächst $=0$ angenommen wird, so muss $\frac{r}{n}$ allmählich zunehmen, bis es schliesslich $=1$ wird $^{1}$ ), und zwar muss die Differenz zweier

1) Dass in einer guten Versuchsreihe $\frac{r}{n}$ bei wachsendem $D$ fortwährend 
Verhältnisse $\frac{r}{n}$, die $2 \mathrm{um}$ eine constante Grösse (z. B. $1 \mathrm{~mm}$ ) verschiedenen Spitzenabständen zugehören, entweder gleichfalls constant bleiben oder um so geringer sein, je weiter die beiden $D$ 's nach oben, bez. nach unten hin von dem Werthe $S$ abstehen, für welchen $\frac{r}{n}=1 / 2$ ist. Es muss also, kurz gesagt, das Verhältniss $\frac{r}{n}$ bei zunehmendem $D$ fortwährend wachsen, bis es schliesslich $=1$ wird, und zwar geht dieses Wachsthum, so lange als $D<S$ ist, mit constanter oder zunehmender Geschwindigkeit, hingegen dann, wenn $D>S$ ist, mit constanter oder abnehmender Geschwindigkeit vor sich. Das Verhältniss $\frac{f}{n}$ ist, falls nur richtige und falsche Fälle erhalten werden, $=1-\frac{r}{n}$, mithin dann, wenn der Gang von $\frac{r}{n}$ ein normaler ist, immer gleichfalls normal ausgefallen. Kommen jedoch auch noch unentschiedene Fälle vor, so ist der normale Gang von $\frac{f}{n}$ folgender: bei wachsendem $D$ nimmt $\frac{f}{n}$ fortwährend $a b$, bis es schliesslich $=0$ wird, und zwar geschieht diese Abnahme, so lange als $D<S$, d. b. kleiner als derjenige Spitzenabstand ist, für welchen $\frac{f}{n}=1 / 2$ ist, mitconstanteroderzunehmender Geschwindigkeit, wenn aber $D>S^{u}$ ist, mit constanter oder abnehmender Geschwindigkeit. Hinsichtlich des Verhältnisses $\frac{z}{n}$, d. h. der relativen Zahl der' unentschiedenen Fälle, ergiebt sich, dass $\frac{z}{n}$ normaler Weise bei wachsendem $D$ entweder constant bleibt oder zunächst gleichfalls wächst, bis es bei einem Werthe von $D$, der zwischen $S^{\prime}$ und $S$ fällt ${ }^{1}$ ), ein Maximum erreicht und dann nach Ueberschreitung dieses Werthes von $D$ allmählich wieder zunehmen müsse, bis es zuletzt $=1$ werde, ist bisher allgemein zugestanden worden.

1) Dieser Werth von $D$ muss genau $=\frac{S^{\prime}+S}{2}$ sein, falls die Wahrscheinlichkeit des Fehlers $\pm \delta$ und $\pm \delta^{\prime}$ eine gerade Funktion der Fehlergrösse ist. 
abnimmt, so dass es schliesslich bei hohen Werthen von $D$ gleich 0 wird.

Nur solche Versuchsrẹihen, in denen der Gang der Verhältnisse $\frac{r}{n}, \frac{f}{n}$ und $\frac{z}{n}$ der im Vorstehenden angedeutete ist, dürfen als normal ausgefallen betrachtet und zu eingehenderen Untersuchungen verwandt werden. Falls es sich nur um eine ungefähre Bestimmung der Feinheitsmaasse des Ortssinnes und der entsprechenden Präcisionsmaasse handelt, kann man allerdings auch solche Versuchsreihen benutzen, deren Resultate die obigen Bedingungen nicht ganz genau erfüllen. Wenn es sich aber um feinere Fragen, z. B. darum handelt, ob das Wahrscheinlichkeitsgesetz der Fehler $\pm \delta$ und $\pm \delta^{*}$ hinlänglich mit dem Gauss'schen Gesetze übereinstimme und mithin die Fundamentalgleichungen (1) bis (4) als hinlänglich zutreffend zu betrachten seien, so wird man nur solche Versuchsreihen berücksichtigen dürfen, die sich dem Obigen gemäss als ganz normal ansgefallene betrachten lassen. Und wir haben in der That gesehen, dass diejenigen der bisherigen Versuchsreihen, welche dieser Bedingung geniigen, unsere Fundamentalgleichungen als hinlänglich gültig erweisen. Obwohl übrigens die Resultate der meisten der bisherigen Versuchsreihen in Folge zu geringer Versuchszahl den obigen Kriterien einer guten Versuchsreihe nicht ganz genau entsprechen, so lässt sich doch bei näherer Vergleichung denselben leicht entnehmen, dass der normale Gang der Verhältnisse $\frac{r}{n}, \frac{f}{n}$ und $\frac{z}{n}$ in der That der oben angedeutete ist.

\section{Historisch-Kritisches.}

§. 13.

Mit wenigen Worten haben wir schliesslich noch auf die bisherigen Verwendungen der erhaltenen Zahlen richtiger und falscher Fälle einzugehen. Kottenkamp und Ullrich (a. a. O. S. 47) gingen von der Voraussetzung aus, dass die von Fechner für ein anderes Untersuchungsgebiet aufgestellte Formel:

$$
\frac{r}{n}=\frac{1}{2}+\frac{1}{\sqrt{\pi}} \int_{0}^{c t} e^{-t^{2}} d t,
$$

wo $t=h D$ gesetzt ist, auch für den Ortssinn der Haut gitltig sei. 
Sie berechneten nach dieser Formel das Präcisionsmaass $h$, in welchem sie ein Feinheitsmaass des Ortssinnes erblickten, indem sie den aus $\frac{r}{n}$ abgeleiteten Werth von $t$ durch den Werth $D$ des benutzten Spitzenabstandes dividirten, also $h=\frac{t}{D}$ setzten. Hiergegen ist erstens zu bemerken, dass das Präcisionsmaass $h$ nicht als Maass der Ortsempfindlichkeit dienen kann, sondern (vgl. §. 2) uns nur über den mittleren Werth der zufälligen Schwankungen der Raumschwelle (der Fehler $\pm \delta$ ) Auskunft gibt. Zweitens ist das aus $\frac{r}{n}$ nach obiger Formel ableitbare $t$ nicht $=h D$, sondern, wie sich aus $\S .2$ ergibt, vielmehr $=h(D-S)$ zu setzen. Indem also Kottenkamp und Ullrich die Feinheit des Ortssinnes gleich $\frac{t}{D}$ setzen, betrachten sie thatsächlich nicht $\frac{h D}{D}$, d. i. $h$, sondern vielmehr $\frac{h(D-S)}{D}$, d. i. $h-\frac{h S}{D}$ oder, wenn wir das für eine und dieselbe Hautstelle bei variablem $D$ constante Produkt $h S$ mit $k$ bezeichnen, $h-\frac{h}{D}$ als Maass der Ortsempfindlichkeit. Wenn sie daher die Ortsempfindlichkeit einer Hautstelle merkwürdiger Weise von $D$ abhängig und zwar um so grösser fanden, je grösser $D$ war, so dass sie sich deshalb veranlasst fanden, diejenigen Werthe von $\frac{r}{n}$, die $<1 / 2$ waren, bei Bestimmung von $h$ gar nicht mit zu berïcksichtigen, so war dies eine ganz natürliche Folge ihres unzutreffenden Verfahrens; denn je beträchtlicher $D$ ist, desto grösser fällt natürlich auch $h-\frac{k}{D}$ aus, wenn die. untersuchte Hautstelle dieselbe bleibt und mithin $k$ constant ist.

\section{§. 14 .}

Der Umstand, dass nach dem von Kottenkamp und U11rich befolgten Verfahren die Ortsempfindlichkeit einer and derselben Hautstelle je nach dem angewandten Spitzenabstande verschieden ausfällt, veranlasste Vierordt (Zeitschr. f. Biol., VI, S. 54 ff.), die Versuchsresultate jener beiden Beobachter einer an- 
deren Behandlung zu unterwerfen. Mit Vierordt's Verfahren stimmt im Wesentlichen auch das von Paulus (a. a. O. S. 246) eingeschlagene, von Riecker und Hartmann späterhin gleichfalls befolgte Verfahren überein. Sehen wir von einigen unwesentlichen Einzelheiten ab, hinsichtlich deren die Verfahrungsweisen dieser vier Forscher nicht gänzlich mit einander übereinstimmen, so ist dieses nene Verfahren kurz folgendes. Es wird von der Voraussetzung ausgegangen, dass die Ortsempfindlichkeiten verschiedener Hautstellen sich umgekehrt zu einander verhalten wie die Spitzenabstände, die an den verschiedenen Hautstellen erforderlich sind, um einen gleichen Werth des Verhältnisses $\frac{r}{n}$ zu ergeben. Demgemäss wird nach den erhaltenen Versuchsresultaten so gut als möglich zu bestimmen gesucht, welcher Spitzenabstände es an den verschiedenen Hautstellen bedürfe, um bestimmte Worthe von $\frac{r}{n}$ $\left(\frac{r}{n}=0,05 ; \frac{r}{n}=0,10 ; \frac{r}{n}=0,15\right.$ u. s. f.) zu erzielen $\left.{ }^{1}\right)$. Auf Grund solcher Bestimmungen wird das relative Feinheitsmaass des Ortssinnes einer Hautstelle, d. h. das Verhältniss, in welchem die Ortsempfindlichkeit derselben zu der $=1$ gesetzten Ortsempfindlichkeit einer bestimmten anderen Hantstelle steht, in der Weise bestimmt, dass man dasselbe dem Verhältnisse reciprok setzt, in welchem der an der ersteren Hautstelle zu Erzielung eines bestimmten $\frac{r}{n}$ erforderliche Spitzenabstand zu dem an der zweiten Stelle zu ganz gleichem Erfolge erforderlichen Spitzenabstande steht. Den verschiedenen Werthen von $\frac{r}{n}$ entsprechend, für welche man an jeder Hautstelle die entsprechenden Spitzenabstände bestimmt hat, erhält man hierbei fuir jede Hautstelle mehrere mehr oder weniger von einander abweichende Werthe des relativen Feinheitsmaasses, aus denen man durch Mittelziehung den endgiiltigen Werth des relativen Maasses der Ortsempfindlichkeit gewinnt.

1) Die graphische Methode, mittels deren Paulus (a. a. o. S. 246), Riecker und Hartmann diese Spitzenabstände bestimmen, bringt aus mehreren Gründen, ebenso wie das entsprechende Verfahren Vieror d t's, Ungenauigkeiten mit sich, die sich andernfalls vermeiden lassen. 
Ebenso wie das Verfahren von Kottenkamp und Ullrich ist auch dieses zweite Verfahren durchaus unzulässig, weil die Voraussetzung eine irrige ist, dass die Spitzenabstände, die erforderlich sind, um an zwei verschiedenen Hautstellen ein gleiches $\frac{r}{n}$ zu ergeben, nur von den Feinheitsmaassen des Ortssinnes beider Hautstellen abhängig seien und uns über das Verhältniss derselben Aufschluss geben könnten. Wie auf S. 191 ff. gesehen, zeigt die Erfahrung, dass die Raumschwelle, d. h. derjenige Spitzenabstand, der eben den Eindruck einer Doppelberïhrung zu Folge hat, für eine und dieselbe Hautstelle ein zufällig schwankender isst. Will man nun trołz dieser zufälligen Schwankungen des Ortssinnes dennoch ein Maass für die:Ortsempfindlichkeiten der versehiedenen Hautstellen aufstellen, so hat man offenbar die Feinheit des Ortssinnes einer Hautgegend nach dem mittleren Werthe $S$ jener zufällig schwankenden Werthe der Raumschwelle zu messen, etwa indem man die Ortsempfindlichkeit einfach $=\frac{1}{S}$ setzt. Ist an 2 Hautstellen der mittlere Werth der Raumschwelle derselbe, aber der Spielraum der zufälligen Schwankungen derselben verschieden, so wird man beiden Stellen dennoch die gleiche Feinheit des Ortssinnes zuschreiben müssen. Denn wollte man derjenigen Hautstelle, an welcher die zufälligen Schwankungen der Raumschwelle ausgiebiger sind, einen feineren Ortssinn beilegen, weil an ihr die Raumschwelle kleinere Einzelwerthe erlangen kann als an der anderen Stelle, so könnte man ihr eben so gut eine geringere Feinheit des Ortssinnes zuschreiben, weil an ihr die zufälligen Einzelwerthe der Raumschwelle anch merklich grösser ausfallen können als an der anderen Stelle. Indessen leuchtet aus diesem Beispiele ein, dass man, um den Thatsachen des Ortssinnes vollständig gerecht zu werden, nicht blos ein Maass der Feinheit des Ortssinnes, sondern auch noch ein Maass der zufälligen Variabilität der Ortsempfindlichkeit aufzustellen hat, d. h. für jede Hautstelle auch einen Werth bestimmen muss, der uns über den mittleren Werth der zufälligen Grössen $\pm \delta$ Aufschluss gibt, um welche die Raumschwelle in den einzelnen Beobachtungsfällen grösser, bez. kleiner ausfällt als ihr Mittelwerth $S$. Dịeser Werth ist das Präcisionsmaass $h$, das, wie auf S. 193 bemerkt, dem mittleren Werthe jener Grössen $\pm \delta$ reciprok ist. 
Wie unschwer zu erkennen und aus den Fundamentalgleichungen (1) und (2) auf S. 193 leicht zu entnehmen, hängt nun die Grösse $D$ des Spitzenabstandes, der erforderlich ist, um ein bestimmtes Verhältniss $\frac{r}{n}$ zu ergeben, nicht blos, wie Vierordt, Paulus u. A. voraussetzen, von der Feinheit, sondern anch von der zufälligen Variabilität der Ortsempfindlichkeit, nicht blos von dem Werthe $S$, sondern auch von dem Präcisionsmaasse $h$ der betreffenden Hautstelle $a b^{1}$ ). Soll an 2 verschiedenen Hautstellen ein und dasselbe $\frac{r}{n}$ erzielt werden, so muss nach jenen Gleichungen das Produkt $(D-S) h$ für beide Stellen einen und denselben Werth $\tau$ besitzen. Bezeichnen wir den an der ersteren Hautstelle zur Erzielung eines bestimmten $\frac{r}{n}$ erforderlichen Spitzenabstand mit $D_{1}$ und den an der zweiten Hautstelle zur Erzielung eines gleichen $\frac{r}{n}$ erforderlichen Spitzenabstand mit $D_{2}$ und verstehen wir unter $h_{1}$ und $S_{1}$ die der ersteren and anter $h_{2}$ and $S_{2}$ die der zweiten Hautstelle entsprechenden Werthe von $h$ und $S$, so muss also nothwendig

$$
\left(D_{1}-S_{1}\right) h_{1}=\left(D_{2}-S_{2}\right) h_{2}=\tau,
$$

1) Wenn Vierord t neuerdings (Grundriss der Physiologie, S. 317) denjenigen minimalen Spitzenabstand, der in allen Fällen den Eindruck einer Doppelberührung zu Folge hat, d, h. denjenigen Werth von $D$, für welchen nach Gleichung (1) auf S. $193 \frac{r}{n}$ einen mit 1 sehr annähernd übereinstimmenden Werth erlangt, zur Bestimmung der Feinheit des Ortssinnes verwandt wissen will, so ist zu bemerken, dass auch dieser Werth von $D$, wie sehr leicht zu erkennen, nicht blos von der Feinheit, sondern auch von der zufälligen Variabilität der Ortsempfindlichkeit abhängt. Nur derjenige Spitzenabstand, welcher $\frac{r}{n}=1 / 2$ ergibt, ist von der zufälligen Variabilität des Ortssinnes ganz unabhängig; denn nach Gleichung (1) ist dieser Werth von $D$ mit dem Werthe Sidentisch. Es ist żu erwähnen, dass Volkmann (a. a. O. S. 49) denjenigen Spitzenabstand, welcher $\frac{r}{n}=\frac{f}{n}=1 / 2$ ergibt, als die wahrscheinlich erkennbare Distanz bezeichnet und für das zweckmässigste Maass der Ortsempfindlichkeit erklärt. Wie jedoch diese Distanz aus den versehiedenen Beobachtungswerthen von $\frac{r}{n}$ abzuleiten sei, hat Volkmann nicht untersucht. 
mithin $D_{1}=\frac{\tau+S_{1} h_{1}}{h_{1}}$ und $D_{2}=\frac{\tau+S_{2} h_{2}}{h_{2}}$ sein.

Hieraus ergibt sich

$$
\frac{D_{1}}{\bar{D}_{2}}=\frac{\left(\tau+S_{1} h_{1}\right) h_{2}}{\left(\tau+S_{2} h_{2}\right) h_{1}}
$$

Aus dieser Gleichung folgt, dass das Verhältniss der Spitzenabstände, die an zwei versehiedenen Hautstellen ein und dasselbe $\frac{r}{n}$ ergeben, keineswegs, wie Vierordt, Paulus u. A. bei ihren Berechnungen der relativen Feinheitsmaasse des Ortssinnes voraussetzen, dem Verhältnisse reciprok ist, in dem die Feinheitsmaasse des Ortssinnes beider Hautstellen (die Werthe $\frac{1}{S_{1}}$ und $\frac{1}{S_{2}}$ ) zu einander stehen, überhaupt nicht blos von der Feinheit, sondern auch von der zufälligen Veränderlichkeit beider Ortsempfindlichkeiten (den Präcisionsmaassen $l_{1}$ und $h_{2}$ ) abhängt und ausserdem auch noch von dem Werthe $r$ abhängig ist, der sich nach Gleichung (1), bez. (2), aus dem zu Grunde gelegten Verhältnisse $\frac{r}{n}$ ergibt. Es sind also alle Werthe, die Vierordt, Paulus, Riecker und Hartmann für die relativen Feinheitsmaasse des Ortssinnes der verschiedenen Hautgegenden berechnet haben, als unrichtig berechnete zu. betrachten; womit nicht gesagt sein soll, dass die Anwendung des richtig begründeten und bewährt gefundenen Verfahrens III betreffs der Feinheit des Ortssinnes aus den bisherigen Versuchsreihen ganz wesentlich andere Resultate gewinnen lassen werde, als jene Forscher abgeleitet haben.

In obiger Gleichung (9) ändert sich der Werth $\tau$ mit dem zu Grunde gelegten Verhältnisse $\frac{r}{n}$, für welches an den beiden verschiedenen Hautstellen die entsprechenden Spitzenabstände zu bestimmen sind, während $h_{1}$ und $S_{1}, h_{2}$ and $S_{2}$ constant bleiben. Mithin muss nach obiger Gleichung das Verhältniss $\frac{D_{1}}{D_{2}}$, d. h. das Verhältniss der Spitzenabstände, die an 2 verschiedenen Hautstellen ein and dasselbe $\frac{r}{n}$ ergeben, je nach dem Werthe dieses $\frac{r}{n}$ versehieden ausfallen und daher auch das Verhältniss der Orts- 
empfindlichkeiten beider Stellen, wenn es, wie Vierordt, Paulus u. A. gethan haben, reciprok zu $\frac{D_{1}}{D_{2}}$. gesetzt wird, je nach dem zu Grunde gelegten $\frac{r}{n}$ verschieden erhalten werden. Ich bemerke kurz, dass die von Vierordt (Zeitschr. für Biologie, VI. S. 60 f.), Riecker (ebendaselbst, IX, S. 100, und X, S. 194), Paulus, (a. a. O. S. 247 und 250 f.) und Hartmann (a.a. 0. S. 88 f.) aufgestellten Tabellen diese Schlussfolgerung vollkommen bestätigen. Verstehen wir z. B. unter den beiden verglichenen Hautstellen die erste und zweite der von Riecker untersuchten Stellen des Unterschenkels, so besitzt das Verhältniss $\frac{D_{1}}{D_{2}}$ nach den eigenen Angaben Rieckers (a. a. 0. IX, S. 100), je nachdem das zu Grunde gelegte $\frac{r}{n}$ gleich 0,$50 ; 0,60 ; 0,70 ; 0,80 ; 0,90$ ist, folgende verschiedene Werthe: 1,70 $(1,64) ; 1,43(1,42) ; 1,23(1,29) ; 1,11(1,24) ; 1,04(1,11)$. Die in Klammern beigefügten Werthe sind diejenigen Werthe, die ich nach Gleichung (9) mittels der nach Verfahren III aus den unmittelbaren Versuchsresultaten Rieckers abgeleiteten Werthe von $h_{1}$ und $S_{1}, h_{2}$ und $S_{2}$ für $\frac{D_{1}}{D_{2}}$ berechnete.

Neuerdings hat Vierordt (Grundriss der Physiologie, S. 317) auf Grund der Versuche von Kottenkamp und U11rich, Paulus, Riecker and Hartmann eine Tabelle aufgestellt, welehe allgemein ergeben soll, wie sich mit zumehmendem $D$ das Verhältniss $\frac{r}{n}$ ändere, in welchem Verhältnisse also ein Spitzenabstand, der ein bestimmtes $\frac{r}{n}$ ergeben habe, geändert werden müsste, um ein bestimmtes anderes $\frac{r}{n}$ zu ergeben. Diese Tabelle, über deren Ableitung nichts Näheres mitgetheilt wird, fusst auf der Voraussetzung, dass die Spitzenabstände, welche bestimmte Werthe von $\frac{r}{n}$ (z. B. $\frac{r}{n}=0,30 ; \frac{r}{n}=0,60 ; \frac{r}{n}=0,90$ ) ergäben, an allen Hautstellen in ganz denselben Verhältnissen zu einander stiinden. Diese Voraussetzung widerspricht aber ganz offenbar der von Vierordt (Zeitsch. f. Biol. VI, S. 58) selbst hervorgehobenen, ganz unleugbaren Thatsache, dass nach den Versuchen von Kottenkamp 
und Ullrich (und, wie ich hinzufüge, auch nach den Versuchen von Paulus, Riecker und Hartmann), an feinfühlenden Stellen die Abstände der 2 berührten Hautpunkte verhältnissmässig stärker wachsen minssen, um eine grössere Zahl richtiger Entscheidungen zu geben, als an minder feinfühlenden." Wie aus den Fundamentalgleichungen (1) und (2) leicht abzuleiten, würde jene Voraussetzung Vierordt's nur dann erfüllt seìn, wenn das Präcisionsmaass $h$ allgemein dem Werthe $S$ reciprok wäre, so dass der Werth des Produktes $S h$ für alle Hautstellen derselbe sei. Allerdings ist nun in der Regel für eine Hautstelle $h$ um so grösser, je kleiner $S$ ist, aber doch nicht so, dass das Produkt $S h$ immer dasselbe bleibt, sondern vielmehr so, dass dieses Produkt mit der untersuchten Hautregion sich ändert und zwar in der Regel um so kleiner ist, je geringer der Werth $S$ ist. So ist z. B. nach den Versuchen von Kottenkamp und Ullrich, wie aus den Angaben in Tabelle III auf S. 216 hervorgeht, das Produkt $S h$ für eine gewisse Stelle des Oberarms (Volarseite, Lokalität $a$ ) $=3,580$, hingegen fuir eine mit feinerem Ortssinne begabte Stelle der Metacarpalregion der Hand $=0,828$. Aus dem Umstande, dass das Produkt $S h$ in der Regel um so kleiner ist, je feiner die Ortsempfindlichkeit ist, erklärt sich, nebenbei bemerkt, die fast paradox erscheinende, schon von Kottenkamp und U11rich (a. a. O. S. 40) constatirte Thatsache, dass das Verhältniss $\frac{r_{0}}{n}$, d. i. die relative Zahl der bei den Vexirversuchen begangenen Irrthimer, in der Regel um so grösser ist, je feiner der Ortssinn ist. Denn je kleiner das Produkt $S h$ ist, desto grösser muss nach Gleichung (7) auf S. 218 wo $(s-d) h$ nach S. 219 gleich $S h$ zu setzen ist, das Verhältniss $\frac{r_{0}}{n}$ ausfallen.

\section{§. 15 .}

Nur anf Eines möchte ich zam Schlusse noch besonders aufmerksam machen, nämlich darauf, dass die von mir vorgeschlagene Verwendung der experimentell erbaltenen Zahlen richtiger und falscher Fälle nicht blos dadurch sich von den bisher üblichen, unzulänglichen Verfahrungsweisen unterscheidet, dass sie auf einer eingehenden mathematischen Analyse beruht und an den zur Zeit vorliegenden Versuchsreihen sich bestätigt und bewährt, sondern 
vor Allem auch den Vorzug besitzt, dass sie eine viel grössere Ausbeutung des vorliegenden Versuchsmaterials, eine viel eingehendere experimentelle Prüfung des Ortssinnes verstattet. Die bisher üblichen Verfahrungsweisen würden, wenn sie triftig wären, uns nur darïber Auskunft gewähren, wie sich die Feinheit des Ortssinnes an verschiedenen Hautstellen, zu verschiedenen Zeiten u. dergl. m. verhält. Unser neues Verfahren dagegen setzt uns nicht blos in Stand, das Verhalten des Werthes $S$ und $S^{\prime}$, d. i. des Mittelwerthes der Schwelle der richtigen, bez. unentschiedenen, Fälle, näher zu studiren und so über das Verhalten der Feinheit des Ortssinnes nähere Auskunft zu erhalten, sondern lässt uns zugleich in den Präcisionsmaassen $h$ und $h^{\prime}$ zwei Werthe gewinnen, die uns iiber das thatsächlich so wichtige Verhalten der zufälligen Variabilität der Ortsempfindlichkeit näher aufklären können. Wie ferner die Thatsache der unentschiedenen Fälle ergibt (vergl. S. $194 \mathrm{ff}$.), ist in vielen Versuchsreihen derjenige Spitzenabstand, welcher eben den Eindruck einer Doppelberïhrung bewirkt, von demjenigen Spitzenabstande, bei welchem eben der Eindruck einer einfachen Berïhrung entsteht, durch ein mehr oder weniger ausgedehntes Intervall geschieden, innerhalb dessen das Urtheil über die Zahl der berührenden Spitzen zurückgehalten wird. Auch über diesen $\mathrm{Z}_{w}$ ischenraum der unentschiedenen Fälle, anf den schon Lichtenfels (Sitzungsber. d. Wiener Akad., math.-naturw. Cl., Bd. VI, S. 341) und insbesondere $\mathrm{Ch}$. Richet (Recherches expérimentales et cliniques sur la sensibilité, Paris 1877, S. 221) näheres Interesse zu lenken versucht haben, vermögen wir nach den bisher uiblichen Verfabrungsweisen nicht die geringste Auskunft zu erhalten. Hingegen wird dieser von Richet als "zone d'indécision" bezeichnete Zwischenraum der unentschiedenen Fälle nach unserer Auffassung einfach durch die Differenz $S-S^{\prime}$ repräsentirt und ist daher nach unserem Verfahren in seiner Abhängigkeit von verschiedenen Umständen leicht zu erforschen.

Man kann die Begriffe eines richtigen, falschen und unentschiedenen Falles verschieden fassen. Man kann z. B. unter einem richtigen Falle einen solchen Fall verstehen, in dem überhaupt der Eindruck einer Doppelberührung entsteht, oder aber auch (ähnlich wie Kottenkamp und Ullrich thaten) nur einen solehen Fall, wo der Eindruck einer Doppelberührung entsteht und zugleich auch 
die Richtung der Verbindungslinie beider Berührungspunkte richtig beurtheilt wird, u. dergl. m. Es ist zu beachten, dass man bei näherer Verwendung der nnmittelbaren Versuchsresultate von den Fundamentalformeln (1) bis (4) ausgehen kann, mag man den Begriff der richtigen und falschen Fälle in dieser oder in jener Weise gefasst haben. Nur darf man auf Grund dieser Formeln nach Verfahren III berechnete Werthe von $S$ und $h$, bez. $S^{\prime}$ und $h^{\prime}$, nicht sämmtlich in eine Linie stellen, wenn ihnen zum Theil verschiedene Definitionen der richtigen und falschen Fälle zu Grunde liegen. Ja es dürfte sogar interessant sein, näher zu untersuchen, wie sich solche verschieden definirte Werthe von $h$ und $S$, bez. $h^{\prime}$ und $S^{\prime}$, an verschiedenen Hautstellen, unter verschiedenen Versuchsumständen $\mathfrak{u}$. dergl. $\mathrm{m}$. zu einander verhalten. Es bedarf keiner weiteren Ausführungen, um erkennen zu lassen, eine wie reiche Aufklärung eine ausgedehnte Anwendung der Methode der richtigen und falschen Fälle, der vorziuglichsten aller psychophysischen Maassmethoden, uns iber den Ortssinn der Haut zu geben vermag, und wie grosses Verdienst sich Vierordt nicht allein durch die Anregung, die er iiberhaupt den Untersuchungen des Ortssinnes der Haut gegeben hat, sondern insbesondere auch durch seine thatkräftige Einführung der Methode der richtigen und falschen Fälle erworben hat. Nur muss man genau wissen; wie man die nach dieser Methode erhaltenen unmittelbaren Versuchsresultate behufs näherer Erforsehung des Ortssinnes zu verwenden hat. Dies hinläglich eingehend und ohne Einm is chung irgend welcher die Theorie des Orstsinnes betreffender Voraus setzungen darzuthun, habe ich in der vorstehenden Abhandlung versucht, deren allzu sehr angeschwollener Umfang leider nicht mehr erlaubt, bei dieser Gelegenheit auch näher auf die Resultate einzugehen, die sich nach unserem Verfahren aus den bisherigen Versuchsreihen ableiten lassen. 